\title{
Synoptic Responses to Mountain Gravity Waves Encountering Directional Critical Levels
}

\author{
ARMel Martin AND FrançOIS LOTT \\ Laboratoire de Météorologie Dynamique du CNRS, Ecole Normale Supérieure, Paris, France
}

(Manuscript received 28 November 2005, in final form 26 June 2006)

\begin{abstract}
A heuristic model is used to study the synoptic response to mountain gravity waves (GWs) absorbed at directional critical levels. The model is a semigeostrophic version of the Eady model for baroclinic instability adapted by Smith to study lee cyclogenesis. The GWs exert a force on the large-scale flow where they encounter directional critical levels. This force is taken into account in the model herein and produces potential vorticity $(\mathrm{PV})$ anomalies in the midtroposphere.

First, the authors consider the case of an idealized mountain range such that the orographic variance is well separated between small- and large-scale contributions. In the absence of tropopause, the PV produced by the GW force has a surface impact that is significant compared to the surface response due to the large scales. For a cold front, the GW force produces a trough over the mountain and a larger-amplitude ridge immediately downstream. It opposes somehow to the response due to the large scales of the mountain range, which is anticyclonic aloft and cyclonic downstream. For a warm front, the GW force produces a ridge over the mountain and a trough downstream; hence it reinforces the response due to the large scales.

Second, the robustness of the previous results is verified by a series of sensitivity tests. The authors change the specifications of the mountain range and of the background flow. They also repeat some experiments by including baroclinic instabilities, or by using the quasigeostrophic approximation. Finally, they consider the case of a small-scale orographic spectrum representative of the Alps.

The significance of the results is discussed in the context of GW parameterization in the general circulation models. The results may also help to interpret the complex PV structures occurring when mountain gravity waves break in a baroclinic environment.
\end{abstract}

\section{Introduction}

The large-scale flow response to the breaking of vertically propagating gravity waves (GWs) has been the subject of many studies during the last 40 years. They follow the seminal works of Eliassen and Palm (1961) and Bretherton (1969), which have shown that mountain GWs induce a net transfer of momentum from the ground toward the atmosphere. The importance of mountain GWs for the atmospheric circulation is now well established. The parameterization of mountain GWs in general circulation models (GCMs) reduces the cold bias these models present near the tropopause in the Northern Hemisphere midlatitudes (Boer et al.

Corresponding author address: Dr. Armel Martin, Laboratoire de Météorologie Dynamique du CNRS, Ecole Normale Supérieure, 24, rue Lhomond, 75235 Paris CEDEX 05, France.

E-mail: martin@Imd.ens.fr
1984; Palmer et al. 1986; McFarlane 1987). More recent parameterizations of subgrid-scale orography (SSO) include trapped lee waves (Miller et al. 1989) and nonlinear low-level flow blocking (Lott and Miller 1997; Scinocca and McFarlane 2000), which both reduce biases in the low-level winds.

Although SSO parameterizations are often evaluated by looking at systematic errors on the zonal mean flow, they also influence nonzonal planetary-scale patterns. For these patterns, it is noteworthy that the SSO parameterizations are not necessarily helpful, unless they permit that the SSO force has a substantial component in the direction perpendicular to the low-level flow (Lott 1999; Webster et al. 2003). This component can have three origins: (i) the anisotropy of the mountains (Phillips 1984; Scinocca and McFarlane 2000; Webster et al. 2003); (ii) the fact that the mean orography in large-scale models does not produce enough vortex compression (Smith 1979; Lott 1999); and (iii) the presence of directional critical levels encountered by the

DOI: $10.1175 / J A S 3873.1$

(C) 2007 American Meteorological Society 
GWs (Shutts 1995). In this last circumstance, the orientation of the force with respect to the background flow is a consequence of the fact that a monochromatic GW encounters a critical level where the background wind is perpendicular to its horizontal wave vector. The $\mathrm{GW}$ is then absorbed, provided that the background flow Richardson number is larger than 0.25 at this altitude (Booker and Bretherton 1967). Since the momentum flux associated with a monochromatic GW is parallel to its horizontal wave vector, aloft an isolated mountain the selective absorption of one GW at each level results in a force perpendicular to the wind at the same altitude. Note that this effect is the result of a vertical shear and is distinct from the effects of horizontal variations in the background flow, which can modify the horizontal wave vector itself (Bühler and McIntyre 2005).

Another large-scale process related to mountains is lee cyclogenesis, for which various dynamical mechanisms were proposed. The local triggering of standing or transient Eady or Rossby edge waves (Smith 1984, 1986; Davis 1997), the low-level modification of preexisting large-scale unstable baroclinic mode (Pierrehumbert 1985; Speranza et al. 1985; Fantini and Davolio 2001), or the cold front distortion by low-level blocking (Messinger and Pierrehumbert 1986; Schär 1990; Gross 1994) are some of these processes. Actually, they are not exclusive, and this variety of theories illustrates the complexity of this problem. Accordingly, few studies have addressed the role of small-scale orography on it.

Nevertheless, as the resolution of regional models increases, the synoptic scales are nowadays rather accurately represented. Besides, quite recently the forecast models began to solve together the synoptic scales and the mesoscale dynamics, including the upper-level GWs and the low-level potential vorticity (PV) banners appearing downstream of the individual mountain peaks (Hoinka et al. 2003; Liniger and Davies 2003; Schär et al. 2003; Flamant et al. 2004; Jiang and Doyle 2004). This recent progress in numerical modeling appeals for a better understanding of the impact of the small-scale dynamics onto the synoptic flow, which is incidentally one of the central objectives of the Mesoscale Alpine Program (MAP; Binder and Schär 1996; Bougeault et al. 2001). It is also in this context that Aebischer and Schär (1998) have suggested that the low-level cyclonic PV generated along the southwestern flank of the Alps could help to trigger lee cyclogenesis.

Although the interaction between mesoscale and synoptic-scale dynamics has been addressed during MAP, by means of either direct field measurements or numerical simulations, relatively few theoretical studies address this problem. It is nevertheless challenging for at least three reasons. First, the net force due to the breaking GWs acts over a domain where horizontal scales correspond to Rossby numbers of order 1 or larger. In this case, the large-scale response to the GWs is not entirely balanced but also contains reemitted inertio-gravity waves (Scavuzzo et al. 1998; Lott 2003). Second, in the midtroposphere the mountain GWs have little chance to break if the wind increases unidirectionally, because the stationary waves' vertical wavenumber decreases with altitude. Nevertheless, in the presence of fronts, many GWs can be absorbed at directional critical levels in the midtroposphere (Shutts 1995) and break thereafter (Broad 1999). The effect of this process on the large-scale flow has never been evaluated. Third, a force that is everywhere perpendicular to the background wind can produce dipolar PV banners with an unusual structure.

This paper presents a theoretical model of the largescale effect of the GWs generated by an idealized front passing over a mountain range. This model accounts for the GWs through the large-scale momentum deposit they induce where they encounter critical levels, in the midtroposphere. For this purpose, we adopt a semigeostrophic (SG) version of Smith's (1984) model of lee cyclogenesis in which we include a GW force following Shutts (1995). The use of a balanced formalism here is supported by Lott (2003), which has shown by direct 2D simulations that after $12 \mathrm{~h}$ typically the balanced part dominates the inertio-gravity waves' part in the total response to GW absorption at a critical level. In this framework, we analyze the surface response associated with the PV anomaly produced by the GW force and compare this response with that due to the smooth large-scale mean orography.

The paper is organized as follows. In section 2 we present the model. In section 3 we analyze the case of a cold front interacting with an idealized complex mountain range in the absence of tropopause. In section 4 we describe the warm front case. In section 5 we compare the quasigeostrophic (QG) response with the SG response, we present some sensitivity tests to the altitude at which the gravity waves interact with the background flow, and we analyze the influence of the tropopause (which introduces unstable baroclinic modes). In section 6 we present the results for orographic spectra representative of the Alps. Section 7 is a summary and a discussion of the implications of our results to the parameterization of mountain GWs in GCMs, or to the interpretation of the PV patterns produced in the midtroposphere when a front crosses a mountain range. 


\section{Model}

a. Equations for the large-scale flow

A central assumption of our model is that the power spectrum of orography shows a clear separation between the large scales and the small scales. This permits us to study separately the synoptic dynamics and the GW dynamics. Although this separation is not valid in reality, it is implicitly assumed in SSO parameterization schemes. For this assumption to be valid, we adopt in sections 3,4 , and 5 an idealized mountain range profile given by

$$
\begin{aligned}
h(\mathbf{x}) & =H_{0} e^{-\left(x^{2}+y^{2} / 2 L^{2}\right)}\left[1+\cos \left(\mathbf{k}_{w} \mathbf{x}\right)\right] \\
& =\mathcal{H}(\mathbf{x})\left[1+\cos \left(\mathbf{k}_{w} \mathbf{x}\right)\right],
\end{aligned}
$$

where $\mathbf{k}_{w}=k_{w} \mathbf{e}_{x}+l_{w} \mathbf{e}_{y}$. In Eq. (1), $L$ is the characteristic large scale, $\mathbf{k}_{w}$ is the dominant horizontal wavenumber associated with the small scales, $2 H_{0}$ is the maximum altitude of the mountain range, and $\mathcal{H}(\mathbf{x})$ is the large-scale orography profile, that is, the envelope of the ridges (Figs. 1a,b).

In the linear context, and in the absence of critical levels, the large-scale effect of the GWs forced by $h^{\prime}=$ $\mathcal{H}(\mathbf{x}) \cos \left(\mathbf{k}_{w} \mathbf{x}\right)$ is very small if $k_{w} L \gg 1$. Nevertheless, if the GWs encounter directional critical levels (Fig. 1c), they are absorbed and can deposit the momentum they transport. For the large-scale flow, this effect can be translated into a force per unit mass (see section $2 \mathrm{c}$ and the appendix),

$$
\mathcal{F}(\mathbf{x}, z)=\mathcal{F}(\mathbf{x}, z) \mathbf{e}_{x}+\mathcal{G}(\mathbf{x}, z) \mathbf{e}_{y},
$$

whose impact adds to that of the large-scale orography profile $\mathcal{H}(\mathbf{x})$.

To study the response to $\mathcal{F}$ and $\mathcal{H}$, we adopt the Boussinesq approximation and consider an idealized front such that the background wind $\mathbf{U}$ and the potential temperature $\Theta_{b}$ have uniform shears,

$$
\begin{gathered}
\mathbf{U}(z)=U(z) \mathbf{e}_{x}+V_{0} \mathbf{e}_{y}=\left(U_{0}+\Lambda z\right) \mathbf{e}_{x}+V_{0} \mathbf{e}_{y}, \\
\Theta_{b}(y, z, t)=\theta_{r}+\theta_{0 z} z+\Theta_{y} y+\theta_{\mathrm{ad}}(t) .
\end{gathered}
$$

In Eqs. (3)-(4), $V_{0}$ is the surface wind, $\Lambda$ is the vertical wind shear, $\theta_{r}$ is a constant reference temperature, $\theta_{0 z}$ is the vertical stratification, $\Theta_{y}$ is the cross-front potential temperature gradient, and $\theta_{\text {ad }}(t)$ is the uniform change in potential temperature associated with the advance of the front. In this framework, the constant BruntVäisälä frequency $N, \Theta_{y}$, and $\theta_{\mathrm{ad}}(t)$ can be written

$$
N^{2}=\frac{g \theta_{0 z}}{\theta_{r}}, \quad \Theta_{y}=-\frac{\Lambda f \theta_{r}}{g}, \quad \text { and } \quad \theta_{\mathrm{ad}}(t)=-V_{0} \Theta_{y} t .
$$

a) $z$

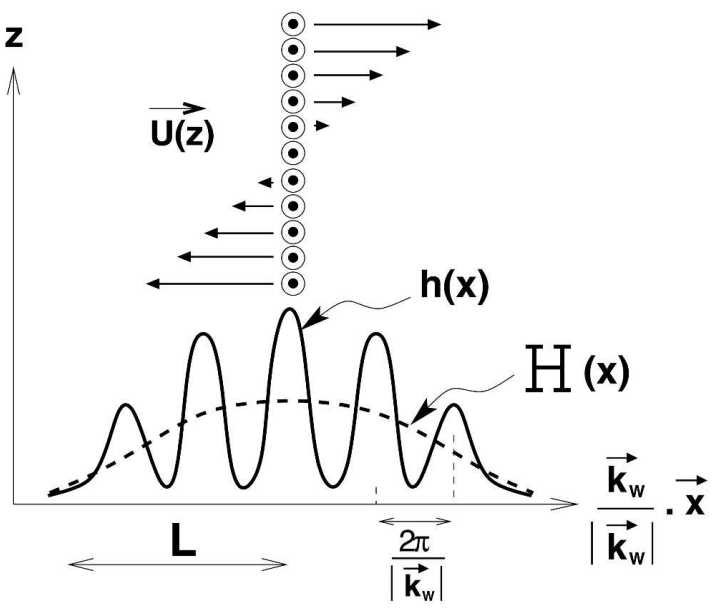

b)

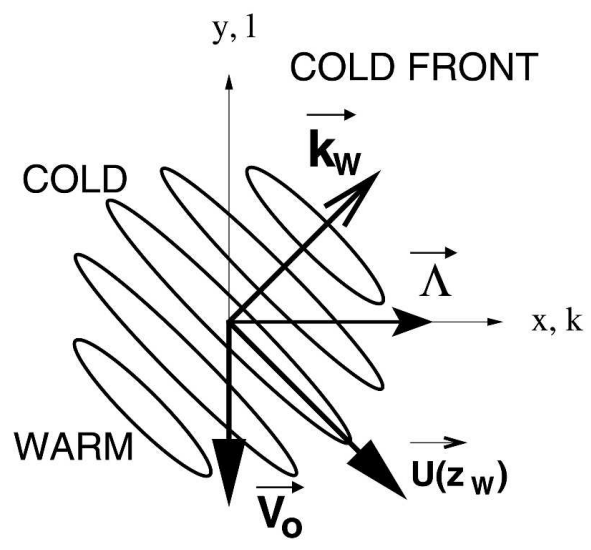

c)

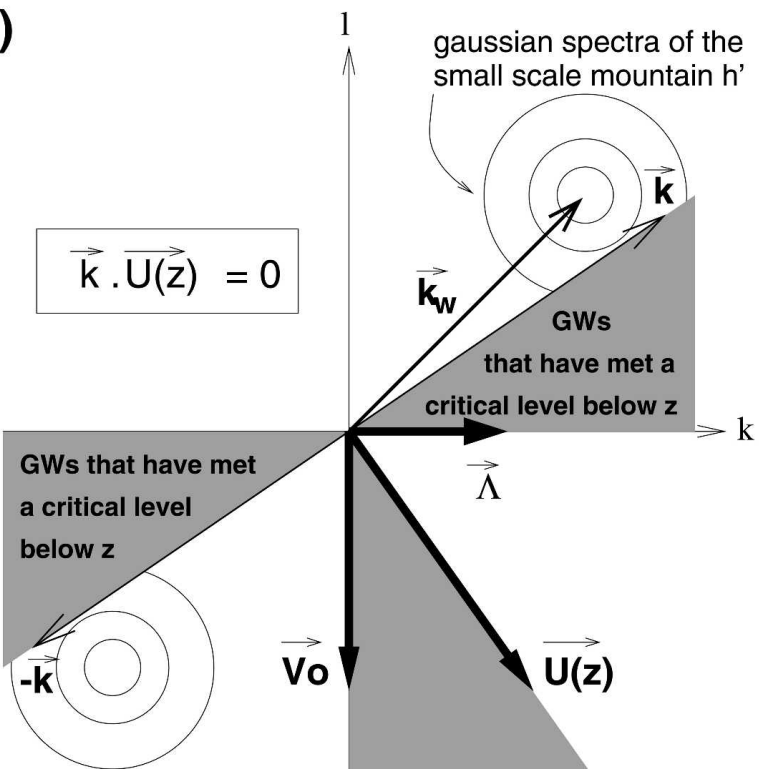

FIG. 1. Schematic representation of the idealized mountain range and of the background flow used to derive the model equations in the cold front case: (a) vertical section with horizontal axis along $\mathbf{k}_{w}$, (b) view from top, and (c) spectrum of the small-scale orography $h^{\prime}=\mathscr{H}(\mathbf{x}) \cos \left(\mathbf{k}_{w} \mathbf{x}\right)$. 
In Eq. (5), the thermal wind balance relates $\Theta_{y}$ to $\Lambda, f$ is a constant Coriolis parameter, and $g$ is the gravity constant.

If we assume that the forcings $\mathcal{F}, \mathcal{G}$, and $\mathcal{H}$ are of small amplitude and have a characteristic horizontal scale $L$ such that the large-scale Rossby number $V_{0} / f L$ is near or below 1 , the response of the flow can be evaluated using a forced and linearized version of the hydrostatic SG equations given in Hoskins (1975):

$$
\begin{aligned}
\left(\partial_{t}+\mathbf{U} \nabla\right) u_{g}+w \Lambda-f v+\partial_{x} \phi & =\mathcal{F}, \\
\left(\partial_{t}+\mathbf{U} \nabla\right) v_{g}+f u+\partial_{y} \phi & =\mathcal{G} \\
\partial_{z} \phi & =g \theta / \theta_{r} \\
\left(\partial_{t}+\mathbf{U} \nabla\right) \theta+v \Theta_{y}+w \theta_{0 z} & =0 \\
\partial_{x} u+\partial_{y} v+\partial_{z} w & =0 .
\end{aligned}
$$

In Eq. (6), $(u, v, w)$ are the components of the wind perturbation, $\phi=p / \rho_{r}$ is the geopotential perturbation, $p$ is the perturbation pressure, and $\rho_{r}$ is a constant reference density. Still in Eq. (6) $u_{g}=\partial_{y} \phi / f$ and $v_{g}=\partial_{x} \phi / f$ are the geostrophic components of the wind perturbation. From the system of Eq. (6), we form the linearized budget of the PV disturbance $q$. For this, we calculate $\{\boldsymbol{\nabla} \times[(6 \mathrm{a}),(6 \mathrm{~b}),(6 \mathrm{c})]\} \cdot \nabla \Theta_{b}+\Lambda \partial_{y}(6 \mathrm{~d})-$ $\theta_{0 z} R_{i}^{-1} \partial_{x}(6 \mathrm{~b})$, and use Eq. (5), which yields

$$
\begin{gathered}
\left(\partial_{t}+\mathbf{U} \boldsymbol{\nabla}\right) \rho_{r} q+\nabla \cdot \mathbf{J}_{N}=0, \text { where } \\
\rho_{r} q(\mathbf{x}, z, t)=\theta_{0 z}\left[\left(1-\mathrm{Ri}^{-1}\right) \partial_{x} v_{g}-\partial_{y} u_{g}\right] \\
\quad+\Lambda \partial_{y} \theta+\Theta_{y} \partial_{z} u_{g}+f \partial_{z} \theta, \text { and } \\
\mathbf{J}_{N}=-\theta_{0 z}\left(1-\mathrm{Ri}^{-1}\right) \mathcal{G} \mathbf{e}_{x}+\theta_{0 z} \mathcal{F} \mathbf{e}_{y} \\
-\Theta_{y} \mathcal{F} \mathbf{e}_{z} .
\end{gathered}
$$

In Eqs. (7) and (9), $\mathbf{J}_{N}$ is the nonadvective PV flux, and $\mathrm{Ri}=N^{2} / \Lambda^{2}$ is the background flow Richardson number. Note that Eqs. (7)-(9) are the linearized version of Hoskins (1975) SG PV budget, with $\mathcal{F}$ added. Notably, $\mathbf{J}_{N}$ is everywhere parallel to the background isentropes $\left(\mathbf{J}_{N} \cdot \nabla \Theta_{b}=0\right)$. And since these isentropes are advected by the background flow, the global PV flux $\rho_{r} q \mathbf{U}(z)+$ $\mathbf{J}_{N}$ never crosses them, which is consistent with the "PV impermeability theorem" (Haynes and McIntyre 1987).

Then, to determine the surface response from the PV, we also consider the two linearized free-slip adiabatic boundary conditions

$$
\begin{aligned}
& {\left[\partial_{t}+\mathbf{U}(0) \nabla\right] \theta+v \Theta_{y}=-\theta_{0 z} \mathbf{U}(0) \nabla \mathcal{H} \quad \text { at } \quad z=0} \\
& {\left[\partial_{t}+\mathbf{U}(D) \nabla\right] \theta+v \Theta_{y}=0 \quad \text { at } \quad z=D .}
\end{aligned}
$$

\section{b. Inversion of the $P V$ perturbation}

If the forcings $\mathcal{F}, \mathcal{G}$, and $\mathcal{H}$ are specified, it is convenient to solve the Eqs. (7)-(11) in the Fourier space. ${ }^{1}$ In this space, the PV perturbation in Eq. (7) is then

$$
\rho_{r} \hat{q}=i \frac{1-e^{i(\mathbf{k} \cdot \mathbf{U}) t}}{\mathbf{k} \cdot \mathbf{U}}\left\{\Theta_{y} \partial_{z} \hat{\mathcal{F}}+\theta_{0 z}\left[-i k\left(1-\mathrm{Ri}^{-1}\right) \hat{\mathcal{G}}+i l \hat{\mathcal{F}}\right]\right\}
$$

provided that $\hat{q}(t=0)=0$. Introducing the geostrophic balance and the hydrostatic relation Eq. (6c), we can invert the PV in terms of geopotential via the elliptic equation

$$
\begin{gathered}
\frac{\partial^{2} \hat{\phi}}{\partial z^{2}}-2 i \lambda_{i} \frac{\partial \hat{\phi}}{\partial z}-\left(\lambda_{r}^{2}+\lambda_{i}^{2}\right) \hat{\phi}=\frac{g}{f \theta_{r}} \rho_{r} \hat{q}, \text { where } \\
\lambda_{r}=\frac{N}{f} \sqrt{1-\mathrm{Ri}^{-1}}|\mathbf{k}| \text { and } \lambda_{i}=l \frac{\Lambda}{f} .
\end{gathered}
$$

First, we form a particular solution that contains the whole PV $\hat{q}$ and vanishes at $z=0$ :

$$
\hat{\phi}_{p}(\mathbf{k}, z, t)=e^{-\lambda z} \int_{0}^{z} e^{2 \lambda_{r} z^{\prime}} \int_{z^{\prime}}^{D}-\frac{g \rho_{r}}{f \theta_{r}} \hat{q} e^{-\lambda^{*} z^{\prime \prime}} d z^{\prime \prime} d z^{\prime},
$$

$$
\text { where } \lambda=\lambda_{r}-i \lambda_{i} \text { and } \lambda^{*}=\lambda_{r}+i \lambda_{i} \text {. }
$$

Then, to satisfy the boundary conditions, we add to this particular solution two boundary waves

$$
\hat{\phi}(\mathbf{k}, z, t)=\hat{\phi}_{p}(\mathbf{k}, z, t)+\hat{\phi}_{u}(\mathbf{k}, t) e^{-\lambda z}+\hat{\phi}_{d}(\mathbf{k}, t) e^{+\lambda^{*}(z-D)}
$$

In this formalism, the boundary conditions Eqs. (10)(11) become

$$
\begin{aligned}
& \lambda_{r}\left[\partial_{t}-i \mathbf{k} \cdot \mathbf{U}(0)\right]\left(\hat{\phi}_{u}-\hat{\phi}_{d} e^{-\lambda^{*} D}\right)-i k \Lambda\left(\hat{\phi}_{u}+\hat{\phi}_{d} e^{-\lambda^{*} D}\right) \\
&=\left\{\left[\partial_{t}-i \mathbf{k} \cdot \mathbf{U}(0)\right] \partial_{z} \hat{\phi}_{p}(0)+\Lambda \hat{\mathcal{F}}(0)\right\}_{W} \\
&-\left\{i \mathbf{k} \cdot \mathbf{U}(0) N^{2}\left(1-\mathrm{Ri}^{-1}\right) \hat{\mathcal{H}}(\mathbf{k})\right\}_{E} \\
& \lambda_{r}\left[\partial_{t}-i \mathbf{k} \cdot \mathbf{U}(D)\right]\left(\hat{\phi}_{u} e^{-\lambda D}-\hat{\phi}_{d}\right)-i k \Lambda\left(\hat{\phi}_{u} e^{-\lambda D}+\hat{\phi}_{d}\right) \\
&=\left\{-\lambda_{r}\left[\partial_{t}-i \mathbf{k} \cdot \mathbf{U}(D)\right] \hat{\phi}_{p}(D)\right. \\
&\left.+i k \Lambda \hat{\phi}_{p}(D)+\Lambda \hat{\mathcal{F}}(D)\right\}_{W}
\end{aligned}
$$

\footnotetext{
${ }^{1}$ Convention: $\phi(\mathbf{x})=\int_{-\infty}^{\infty} \int_{-\infty}^{\infty} \hat{\phi}(\mathbf{k}) e^{-\mathrm{ikx}} d k d l$, where $\hat{\phi}(\mathbf{k})=$ $\left(1 / 4 \pi^{2}\right) \int_{-\infty}^{\infty} \int_{-\infty}^{\infty} \phi(\mathbf{x}) e^{i \mathbf{k x}} d x d y$.
} 
at $z=0$ and $z=D$, respectively. In the right-hand side of Eqs. (18)-(19) we have separated the forcings due to the GWs $(W)$, and to the envelope $\hat{\mathcal{H}}(E)$. In the following, the response is always described at the ground, where $\hat{\phi}=\hat{\phi}_{W}+\hat{\phi}_{E}$ because $\hat{\phi}_{p}(z=0)=0$.

\section{c. Evaluation of $\mathcal{F}(x, z)$}

To evaluate the force $\mathcal{F}(\mathbf{x}, z)$, we first calculate a net force $\overline{\mathcal{F}}(z)$ using the linear theory of GWs in a linear background shear flow (Shutts 1995 and the appendix):

$$
\begin{aligned}
& \overline{\mathcal{F}}(z)=-\frac{d}{d z} \overline{\mathbf{u}^{\prime} w^{\prime}}, \quad \text { where } \\
& \overline{\mathbf{u}^{\prime} w^{\prime}} \equiv \frac{1}{\pi L^{2}} \int_{-\infty}^{+\infty} \int_{-\infty}^{+\infty} \mathbf{u}^{\prime} w^{\prime} d x d y .
\end{aligned}
$$

In Eq. (20), $\mathbf{u}^{\prime}$ and $w^{\prime}$ are the small-scale velocities associated with $h^{\prime}$. Then, we consider that the horizontal distribution of $\mathcal{F}(\mathbf{x}, z)$ resembles the envelope profile $\mathcal{H}(\mathbf{x})$ :

$$
\mathcal{F}(\mathbf{x}, z)=\overline{\mathcal{F}}(z) e^{-\left(x^{2}+y^{2}\right) / L^{2}} .
$$

Although in Eq. (21), $\mathcal{F}(\mathbf{x}, z)$ results in a net force equal to $\overline{\mathcal{F}}(z)$, Eq. (21) assumes that the GW field stays confined over the mountain range $\mathcal{H}$. This assumption is only valid for hydrostatic waves in the absence of shear, while for the background flow we consider here, Shutts and Gadian (1999) have shown for an isolated mountain that the GW field is spread downstream over a distance that can reach a few times the horizontal scale of the mountain. Nevertheless, we adopt this assumption for several reasons. First, the drag $\overline{\mathbf{u}^{\prime} w^{\prime}}$ is less widely spread because it is quadratic. Second, Lott (2003) have shown in 2D that the spread of the drag is reduced if we increase the vertical resolution and add a small amount of dissipation to take account of the breaking. Third, since we consider here the combined effect of several different anisotropic individual ridges, a possible local shift of the force over a distance comparable to $2 \pi /\left|\mathbf{k}_{w}\right|$ can be reasonably neglected in respect to $L$, the scale of the global mountain.

As our GWs have horizontal wavenumber $\mathbf{k} \sim \pm \mathbf{k}_{w}$ [Eq. (1) and Figs. 1b,c], the forcing $\mathcal{F}$ is only significant if there exists a critical level $z_{w}$ for $\mathbf{k}_{w}: \mathbf{U}\left(z_{w}\right) \cdot \mathbf{k}_{w}=0$. In other words, the background wind has to pass from one side of the ridges to the other when $z$ increases (Fig. 1b). For a cold front in the Northern Hemisphere, for example, $V_{0}<0$ and $\Lambda>0$, this only occurs if $k_{w} \cdot l_{w}>0$ (Figs. $1 \mathrm{~b}, \mathrm{c}$ ). For a warm front $\left(V_{0}>0\right.$ and $\Lambda>0$ ) this only occurs if $k_{w} \cdot l_{w}<0$. In both cases, the total force is well approximated by

$$
\begin{aligned}
\overline{\mathcal{F}}(z) \approx & \frac{1}{2 \sqrt{\pi}} N \Lambda L H_{0}^{2} k_{0}(z)^{2} e^{-L^{2}\left\|\mathbf{k}_{w}\right\|^{2}+L^{\prime 2} k_{0}^{2}} \frac{\Lambda z\left|V_{0}\right|}{\|\mathbf{U}(z)\|^{2}} \\
& \times\left(\mathbf{e}_{x}-\frac{U(z)}{V_{0}} \mathbf{e}_{y}\right),
\end{aligned}
$$

where

$$
\begin{aligned}
k_{0} & =\left[k_{w}-l_{w} \frac{U(z)}{V_{0}}\right] /\left[1+\frac{U(z)^{2}}{V_{0}^{2}}\right], \text { and } \\
L^{\prime 2} & =\left[1+\frac{U(z)^{2}}{V_{0}^{2}}\right] L^{2} .
\end{aligned}
$$

Coherently, $\mathcal{F}(z)$ is everywhere orthogonal to the background flow $\mathbf{U}(z)$ (see the appendix).

\section{d. Computation}

To solve this problem numerically, we consider a horizontally periodic domain of size $20000 \mathrm{~km} \times$ $20000 \mathrm{~km} \times 10 \mathrm{~km}$. The fields are represented by $512 \times 512$ harmonics and 81 vertical levels, yielding a resolution of $40 \mathrm{~km} \times 40 \mathrm{~km} \times 125 \mathrm{~m}$. First, we compute analytically the PV anomaly using the diagnostic Eq. (12) and integrate it vertically by a trapezoidal approximation to evaluate $\hat{\phi}_{p}$ in Eq. (15). Then, we solve the first-order differential Eqs. (18)-(19), treating separately the parts "W" and "E." This yields temporal integrals that converge for a time step of $2 \mathrm{~h}$.

\section{Cold front with no tropopause}

In this section, we place the tropopause altitude at $D=\infty$. And we consider an idealized cold front moving toward the south in the Northern Hemisphere midlatitudes, across an idealized mountain range. The flow and orography parameters are, respectively,

$$
\begin{aligned}
\theta_{r} & =300 \mathrm{~K}, \quad \rho_{r}=1 \mathrm{~kg} \mathrm{~m}^{-3}, \quad f=10^{-4} \mathrm{~s}^{-1}, \\
N & =10^{-2} \mathrm{~s}^{-1}, \quad \Lambda=4.10^{-3} \mathrm{~s}^{-1}, \\
U_{0} & =0 \mathrm{~m} \mathrm{~s}^{-1}, \quad V_{0}=-20 \mathrm{~m} \mathrm{~s}^{-1} . \\
H_{0} & =800 \mathrm{~m}, \quad L=200 \mathrm{~km}, \\
\left|\mathbf{k}_{W}\right| & =\frac{2 \pi}{70000} \mathrm{~m}^{-1}, \quad \text { with } \quad k_{w}=l_{w} .
\end{aligned}
$$

The mountain range half-height width is $2 L \sqrt{\ln 2} \approx$ $330 \mathrm{~km}$. It is typically constituted of five to seven ridges, which are $70 \mathrm{~km}$ wide and oriented southeastnorthwest (Fig. 1b). The dimensionless mountain heights $N H_{0} / f L=0.4<1$ for the large-scale orography $\mathcal{H}$, and $N 2 H_{0} / V_{0}=0.8<1$ for the small-scale orography $h^{\prime}$, justify a linear treatment for both. The large-scale Rossby number is $V_{0} / f L \approx 1$, and the ratio between the 


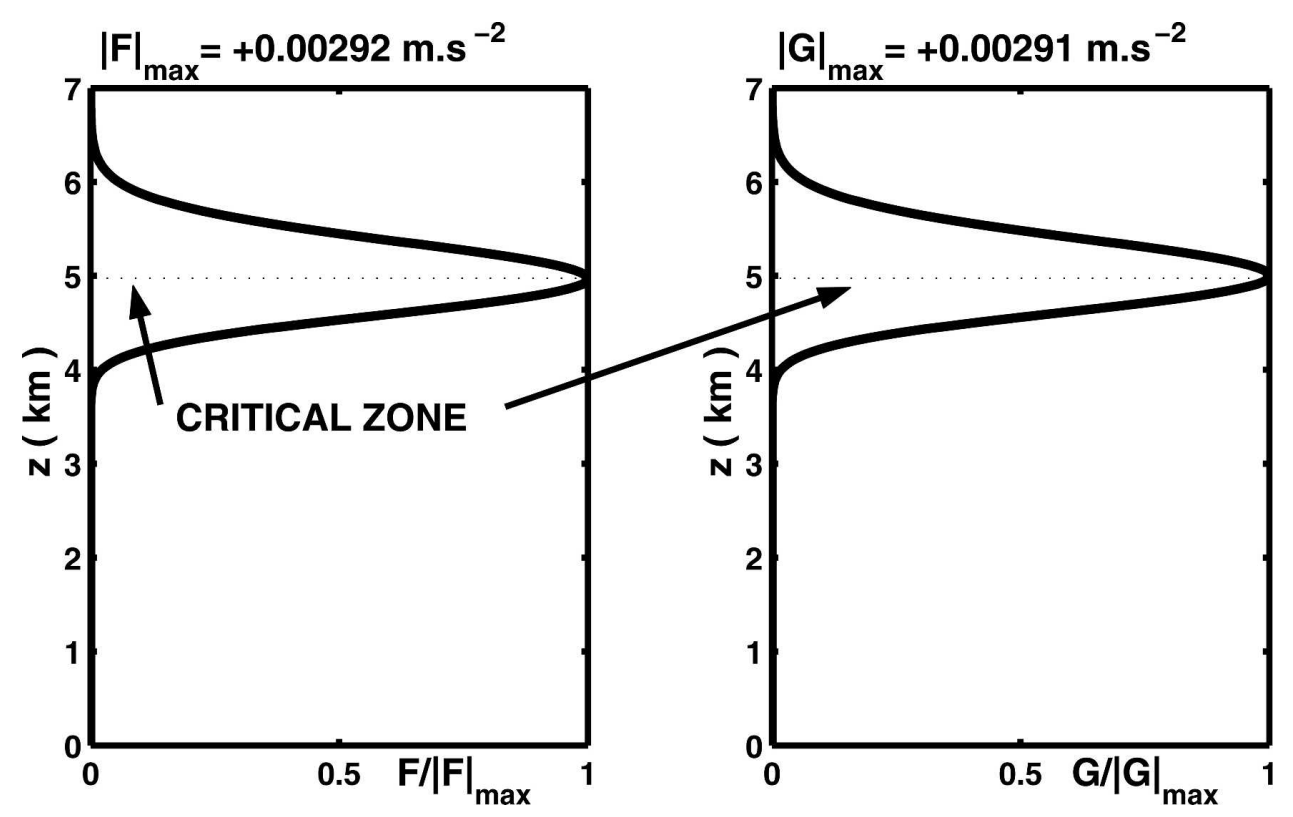

FIG. 2. Vertical profile of the force $\overline{\mathcal{F}}(z)$ in the idealized cold front case in section 3: $\theta_{r}=300 \mathrm{~K}$, $f=10^{-4} \mathrm{~s}^{-1}, N=10^{-2} \mathrm{~s}^{-1}, \Lambda=4.10^{-3} \mathrm{~s}^{-1}, U_{0}=0 \mathrm{~m} \mathrm{~s}^{-1}, V_{0}=-20 \mathrm{~m} \mathrm{~s}^{-1}, H_{0}=800 \mathrm{~m}, L=200 \mathrm{~km}$, $\left|\mathbf{k}_{W}\right|=2 \pi / 70000 \mathrm{~m}^{-1}$, and $k_{w}=l_{w}$.

large and the short scales is $2 L\left|\mathbf{k}_{w}\right| / 2 \pi \approx 6>1$. Note that we could arbitrarily choose to have a bigger number of thinner ridges in the same envelope as here. But, therefore, we should have also reduced the height of these ridges to conserve a reasonable orographic variance and force.

\section{a. $P V$ anomaly due to the absorbed $G W$ forcing $\mathcal{F}$}

The vertical profile of the net force $\overline{\mathcal{F}}(z)$ in Eq. (22) is displayed in Fig. 2. Each component reaches a maximum near $z_{w}=5 \mathrm{~km}$ and is only substantial over a vertical depth of 1 or $2 \mathrm{~km}$ around $z_{w}$. In the rest of the paper we will refer to this area as the critical zone.

Figures $3 \mathrm{a}-\mathrm{c}$ show three horizontal sections of the PV anomaly due to $\mathcal{F}$ in the critical zone at $t=12 \mathrm{~h}$. At the three levels, the PV amplitude is between 0.4 and 0.8 potential vorticity units (PVU; where $1 \mathrm{PVU}=1.0 \times$ $10^{-6} \mathrm{~m}^{2} \mathrm{~s}^{-1} \mathrm{~K} \mathrm{~kg}^{-1}$ ), and the PV patterns are predominantly oriented in the direction of the background wind $\mathbf{U}\left(z_{w}\right)$. As we explain further hereafter, the PV patterns have this structure because first the PV is produced by $\mathcal{F}$ aloft the mountain, and then the $\mathrm{PV}$ is advected by the background flow. To interpret the differences between those three levels, one visualizes schematically the nonadvective PV flux $\mathbf{J}_{N}$ in Fig. 4. Figure 4a shows that $\mathbf{J}_{N}$ is pointing upward to be parallel to the isentropes. In the $(y, z)$ plane, $J_{N z}$ essentially takes the PV from below $z_{w}$ to put it above (Fig. 4a), yielding the predominantly positive PV lobe above $z_{w}$ (Fig. 3a) and the predominantly negative one below (Fig. 3c). Near the altitude $z_{w}$, the divergence of the vertical component of the nonadvective PV flux is null,

$$
\partial_{z} J_{N z}=\Theta_{y} \partial_{z} \mathcal{F}=0 ;
$$

therefore the PV is only due to the vector $\mathbf{J}_{N x y}=J_{N x} \mathbf{e}_{x}+$ $J_{N y} \mathbf{e}_{y}$, which is nearly opposite to the wind, the force $\mathcal{F}\left(z_{w}\right)$ being perpendicular to it (Fig. 4b). Hence, near $z_{w}, \mathbf{J}_{N x y}$ tends to take PV from downstream to put it upstream, according to the direction of $\mathbf{U}\left(z_{w}\right)$. At small times, it results in a positive PV anomaly along the upstream flank of the mountain and a negative one downstream (Fig. 4b). Afterward, the negative PV anomaly produced aloft of the mountain is advected downstream at the velocity $\mathbf{U}\left(z_{w}\right)$, yielding the midtropospheric start-up anticyclone in Fig. 3b. Still near $z_{w}$ but over the mountain, the long-term response is a steady-state cyclonic PV anomaly (Fig. 3b). There, the PV due to $\mathbf{J}_{N x y}$ is exactly balanced by the advection term

$$
(\mathbf{U} \cdot \boldsymbol{\nabla}) \rho_{r} q+\boldsymbol{\nabla} \cdot \mathbf{J}_{N x y}=0 .
$$

The PV structure in Fig. 3 essentially represents a PV banner, whose characteristic horizontal width and depth are fixed by the width and depth of the critical zone, around $2 L=400$ and $2 \mathrm{~km}$, respectively (see Figs. 2 and 4), while its length is controlled by the advection [i.e., around $\left.\left|\mathbf{U}\left(z_{w}\right)\right| t\right]$. This PV banner has a 3D structure that is very different from the PV banners occurring in barotropic flows when the low-level flow passes 


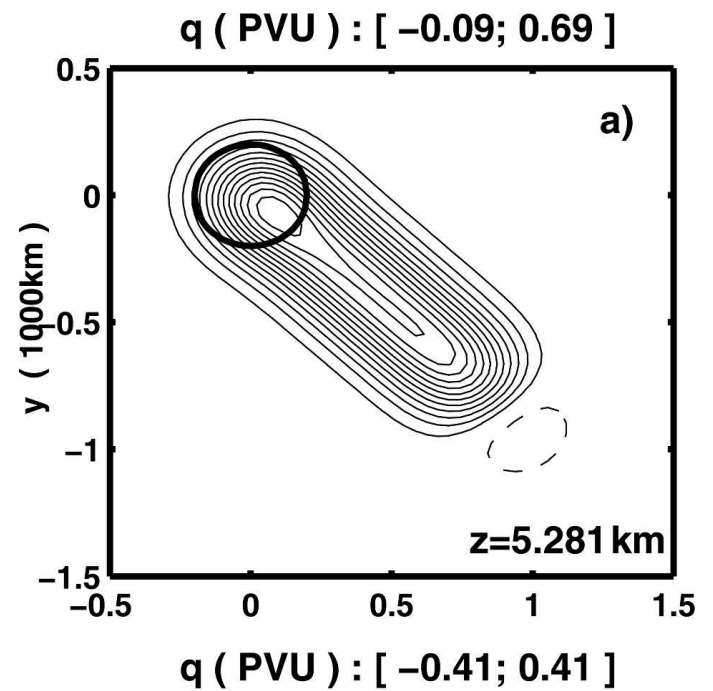

around individual mountain peaks or when mountain GWs break in the absence of critical levels. In these cases, the effect of the mountain can still be modeled by a nonadvective PV flux $\mathbf{J}_{N}$. It is nevertheless perpendicular to the background wind rather than parallel to it, resulting in PV lobes of opposite signs localized one next to the other in the horizontal (Schär and Durrän 1997).

\section{b. Surface pressure $\phi_{W}$ due to the forcing $\mathcal{F}$}

Figures $5 \mathrm{a}-\mathrm{c}$ show the surface pressure perturbation induced by the GW momentum deposit. At small times (Fig. 5a), it presents a small-amplitude (approximately $-0.1 \mathrm{mb}$ ) trough over the mountain and a ridge downstream of it [toward $\mathbf{U}\left(z_{w}\right)$ ]; its amplitude is around 0.2 $\mathrm{mb}$. Over a longer time (Figs. 5b,c), the trough over the mountain near disappears, while the downstream ridge extends along the direction of $\mathbf{U}\left(z_{w}\right)$. To the west of this extending ridge, a second trough is developing (Figs. $5 b, c)$. At $36 \mathrm{~h}$, the maximum amplitude of the surface signal is $1.1 \mathrm{mb}$.

To interpret this response, Fig. 6 shows the surface temperature anomaly $\left(\theta_{r} / g\right) \partial_{z} \phi_{p}(0)$ associated with the particular solution $\phi_{p}$. This term is central because it translates the PV into Eady waves forcing [see Eq. (18)] via the weighted average (in the Fourier space):

$$
\frac{\theta_{r}}{g} \partial_{z} \hat{\phi}_{p}(\mathbf{k}, z=0, t)=-\int_{0}^{D} \frac{\hat{q}}{f} e^{-\lambda^{*} z} d z .
$$

At $t=12 \mathrm{~h},\left(\theta_{r} / g\right) \partial_{z} \phi_{p}(0)$ is negative over the mountain and positive downstream of it. This follows that the midtroposphere PV in Fig. 3 is predominantly positive over the mountain and negative downstream of it. Note also that the positive lobe in surface temperature exceeds in amplitude the negative one, because the negative PV in Fig. $3 \mathrm{c}$ has a stronger influence at the surface than the positive one in Fig. 3a. Note also that the pattern of $\left(\theta_{r} / g\right) \partial_{z} \phi_{p}(0)$ is substantially larger in horizontal size than the PV pattern. Again, this is due to the weight $e^{-\lambda_{r} z}$ in Eq. (28), which favors the large scales, a general property of PV inversion operators (Hoskins et al. 1985). Still at $t=12 \mathrm{~h}$, the surface trough over the mountain and the downstream ridge in Fig. 5a are essentially needed for the surface temperature $\left(\theta_{r} / g\right) \partial_{z} \phi_{W}(0)$ to oppose $\left(\theta_{r} / g\right) \partial_{z} \phi_{p}(0)$.

At later stages $(t=24 \mathrm{~h}, 36 \mathrm{~h})$, the temperature

FIG. 3. Horizontal sections of the PV anomaly in the critical zone, at $t=12 \mathrm{~h}$ and for different heights: (a) $z=5.281 \mathrm{~km}$, (b) $z=4.969 \mathrm{~km}$, and (c) $z=4.656 \mathrm{~km}$. Same parameters as in Fig. 2. Contour interval $(\mathrm{CI})=0.05$ PVU. Negative values are dashed. The minimum and maximum values of $q$ are indicated above each panel. The location and half-height width of the mountain range is indicated by the circle. pattern $\left(\theta_{r} / g\right) \partial_{z} \phi_{p}(0)$ is more complex (Figs. 6b,c), with an elongated negative anomaly appearing along the northeast flank of the extending downstream positive anomaly. This negative anomaly is the surface signature of the upper-level positive PV (the one shown in Fig. 3a at $t=12 \mathrm{~h}$ ). At these times, it is no longer masked by 

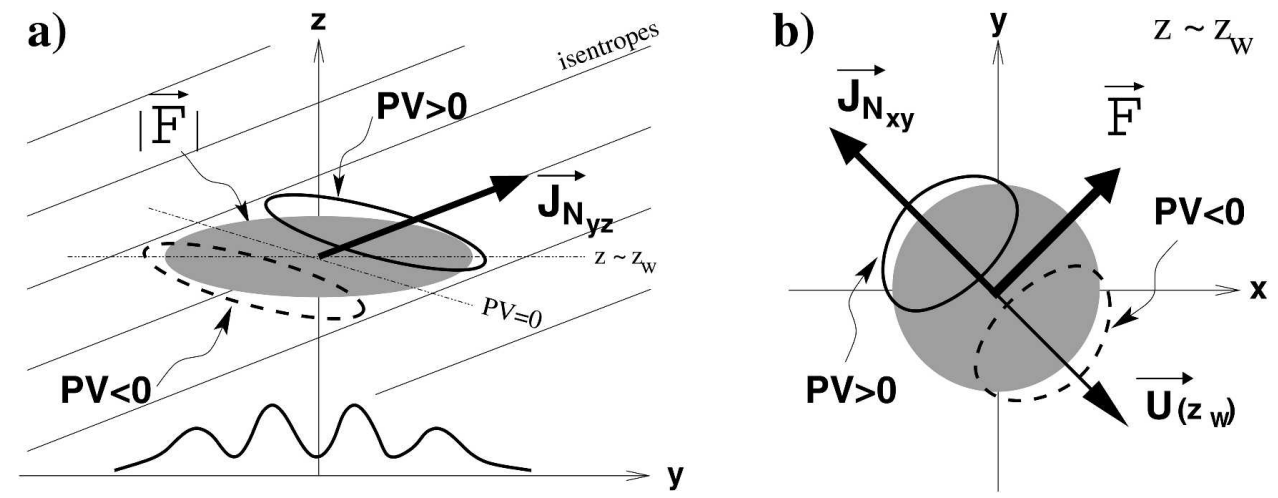

FIG. 4. Schematic representation of the nonadvective PV flux $\mathbf{J}_{N}$ and of its effects on the PV. The PV lobes are schematized by the thick solid and dashed closed lines. The gray area represents the critical zone; that is, the zone where the GWs force $\mathcal{F}$ is substantial. (a) Vertical section at $x=0$; the tilted isentropes are also schematized by thin solid lines, and (b) horizontal section at $z=z_{w}$.

the lower-level negative anomaly (the one in Fig. 3c at $t=12 \mathrm{~h}$ ), because the wind is turning clockwise with altitude.

As a result, the surface response $\phi_{W}$ is predominantly anticyclonic along the axis of $\mathbf{U}\left(z_{w}\right)$ (Figs. 5b,c). Nevertheless, it presents a trough along the southeastern flank of this ridge, to the opposite of the place where the elongated negative surface anomaly of $\left(\theta_{r} / g\right) \partial_{z} \phi_{p}(0)$ is located in Figs. 6 b,c. This bears witness to the fact that boundary Eady waves start to modulate the surface response via the meridional thermal advection in Eq. (18) and make it differ from the simple mirror image of the PV deposit.

To support this last statement, we note that the Eady wave forcing $\left(\theta_{r} / g\right) \partial_{z} \phi_{p}(0)$ (Fig. 6) has a quite stationary structure upstream of the advected start-up anticyclone. In terms of Eady wave dispersion relationships, this translates into

$$
\omega=\mathbf{k} \cdot \mathbf{U}\left(z=\frac{1}{\lambda_{r}}\right)=k \frac{\Lambda}{\lambda_{r}}+l V_{0}=0 .
$$

As the shape of $\left(\theta_{r} / g\right) \partial_{z} \phi_{p}(0)$ also imposes $k \sim l$, Eq. (29) yields $\lambda_{r} \sim \Lambda /-V_{0}=2 \times 10^{-4} \mathrm{~m}^{-1}$ and a dominant wavelength $2 \pi /|\mathbf{k}| \sim 3000 \mathrm{~km}$ rather consistent with Fig. 5c. For these scales, the group velocity points toward the south-southwest:

$$
\mathbf{C}_{g}=\left(\frac{\Lambda}{\lambda_{r}} \frac{l^{2}}{|\mathbf{k}|^{2}}, \frac{\Lambda}{\lambda_{r}} \frac{l^{2}}{|\mathbf{k}|^{2}}\right) \sim\left(-\frac{V_{0}}{2}, \frac{3 V_{0}}{2}\right),
$$

whereas $\mathbf{U}\left(z_{w}\right)$ is southwest. Accordingly, we observe the development of a trough at the west of the extending ridge.

\section{c. Surface pressure $\phi_{E}$ due to $\mathcal{H}(x)$}

The time evolution of $\phi_{E}$ is shown in Figs. $5 \mathrm{~d}-\mathrm{f}$ at $t=$ 12,24 , and $36 \mathrm{~h}$, respectively. A boundary Eady lee wave is developing and extending downwind according to $\mathbf{U}(0)=V_{0} \mathbf{e}_{y}$. The wave is bounded downstream by the start-up cyclone due to the warm potential temperature anomaly $\theta_{0 z} \mathcal{H}$ present over the mountain range when $t<0$ and swept away by $\mathbf{U}(0)$ when $t>0$. It is bounded over the mountain by an anticyclonic pattern due to vortex compression. The corresponding high keeps almost constant after $12 \mathrm{~h}$ and reaches $1.2 \mathrm{mb}$ at $36 \mathrm{~h}$. Immediately downstream of the mountain a trough, whose amplitude reaches $-1.3 \mathrm{mb}$ at $36 \mathrm{~h}$ is settling. The amplitudes obtained are significantly smaller than in Smith $(1984,1986)$ because our mountain is substantially smaller in width and height.

As in section $3 \mathrm{~b}$ this surface response can also be interpreted in terms of stationary boundary Eady wave dynamics. In this case the large-scale orography induces a steady forcing with dominant wavenumbers $|\mathbf{k}| \approx 1 / L$, and Eq. (29) implies

$$
l / k=-\Lambda /\left(\lambda_{r} V_{0}\right) \sim 0.5>0 .
$$

Accordingly, the troughs and ridges are oriented from northwest to southeast and are strongly inclined along the $y$ axis. Likewise, Eq. (31) permits us to predict $2 \pi / l \approx 4 \pi L=2400 \mathrm{~km}$, which roughly corresponds to the crest-to-crest distance along the $y$ axis in Fig. 5f. These values yield a dominant group velocity consistent with the Eady lee wave extension along the $y$ axis in Fig. 5f:

$$
\mathbf{C}_{g} \approx(6,-20) \mathrm{m} \mathrm{s}^{-1}
$$

\section{d. Total response at the surface $\phi=\phi_{W}+\phi_{E}$}

The total response $\phi=\phi_{W}+\phi_{E}$ is displayed in Figs. 5g-i. Although the GW effect is rather small, 

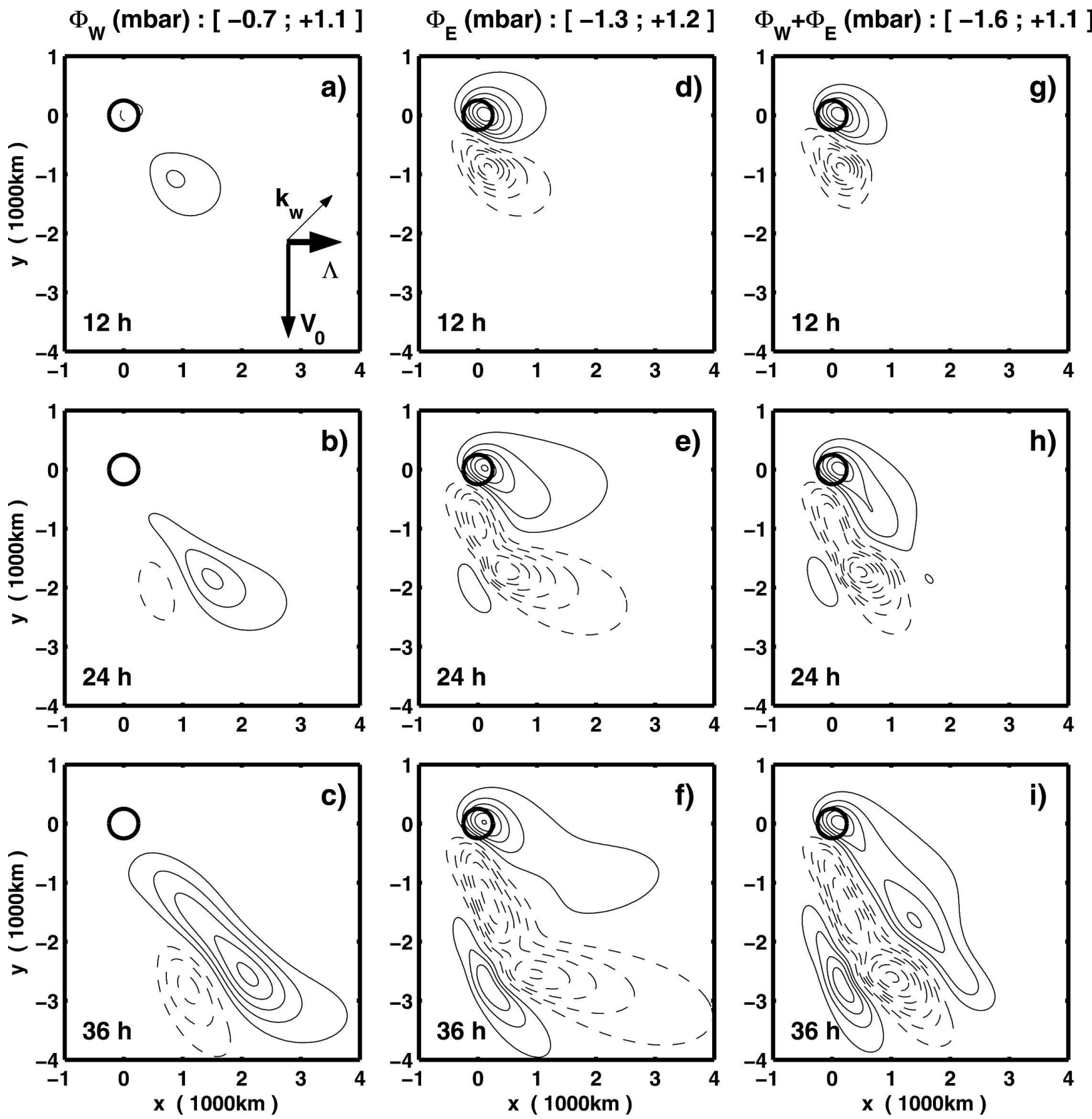

FIG. 5. Evolution of the surface geopotentials at $t=12,24$, and 36 h. Same parameters as in Fig. 2. (a)-(c) Geopotential due to the GWs $\left(\phi_{W}\right)$; (d)-(f) geopotential due to the large-scale orography profile $\mathcal{H}(\mathbf{x})\left(\phi_{E}\right)$; (g)-(i) total perturbation $\left(\phi_{W}+\phi_{E}\right)$. The magnitude is indicated as a pressure in millibars because $\rho_{r}=1 \mathrm{~kg} \mathrm{~m}^{-3}$. Negative values are dashed and CI $=0.2 \mathrm{mb}$. The minimum and maximum values reached at $t=36 \mathrm{~h}$ are indicated at the top of each column. The configuration of the background front with regards to the orientation of the ridges is schematized in (a).

they reduce by near $20 \%$ the ridge produced by the large-scale orography over the mountain, as well as the downstream trough. More pronounced effects occur farther downstream. In particular the ridge produced by the GWs and, which extends below the PV banner in Fig. 5b, substantially affects the surface response in the far field.

\section{Warm front with no tropopause}

In this part, we consider the case of a warm front by taking $V_{0}=20 \mathrm{~m} \mathrm{~s}^{-1}$ in our model. To obtain a significant force $\mathcal{F}$ we also rotate the small-scale ridges and take $k_{w}=-l_{w}$ (see appendix). All the other parameters are the same as in section 3 . 
$\theta_{r} / g \partial_{z} \Phi_{p}(0)(K):[-0.23 ;+0.48]$
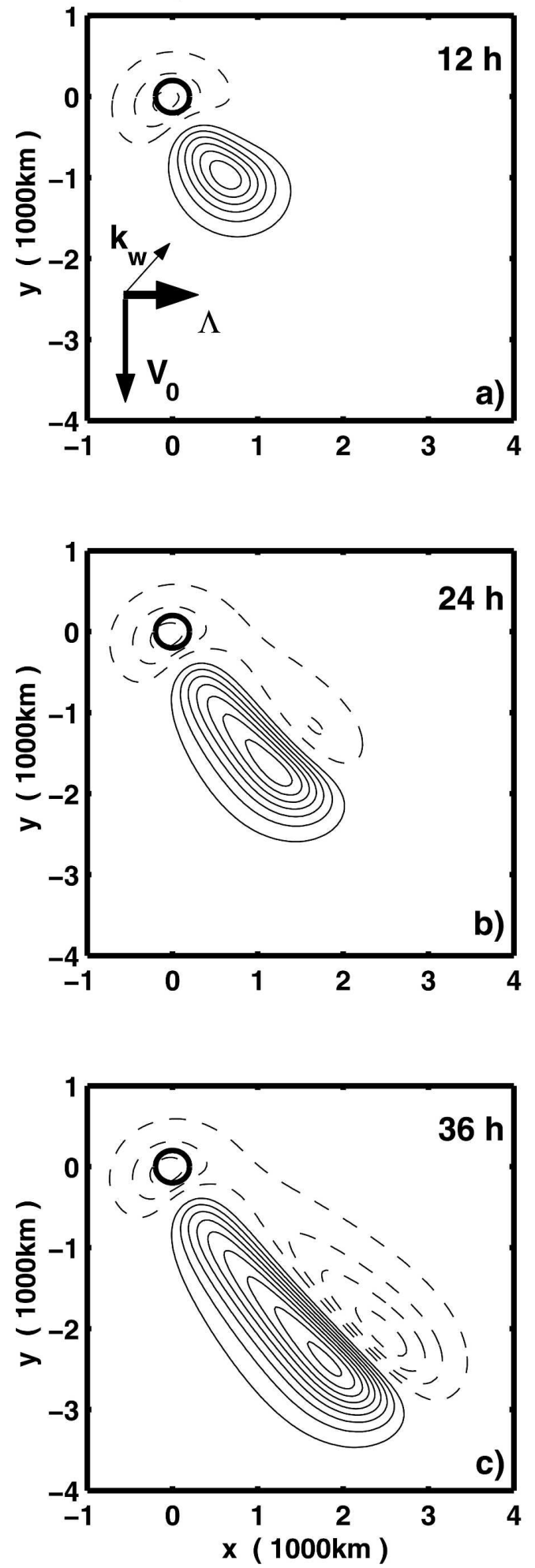

FIG. 6. Evolution of the potential temperature anomaly at the surface $\left(\theta_{r} / g\right) \partial_{z} \phi_{p}(0)$, same parameters as in Fig. 2; (a) $t=12 \mathrm{~h}$, (b) $t=24 \mathrm{~h}$, and (c) $t=36 \mathrm{~h}$. Negative values are dashed and $\mathrm{CI}=$ $0.5 \mathrm{~K}$.
As in the cold front case, the PV anomaly on both flanks of the critical zone has an elongated structure. It is predominantly positive above $z_{w}$ and negative below (Figs. 7a,c). This is almost as in the cold front case in Figs. 3a,c. Near the altitude $z_{w}$ nevertheless (Fig. 7b), the PV anomaly over the mountain is negative, and an opposing pattern of positive PV is advected downstream at the velocity $\mathbf{U}\left(z_{w}\right)$, the opposite to what occurs in the cold front case in Fig. 3b.

This last difference between the cold front case and the warm front case is an important result that has a simple dynamical origin. The necessity that there exist critical levels in the troposphere for the GWs to affect the large-scale flow imposes that the GW force has one component in the direction of the shear $\Lambda$ (the $x$ component in our case, see Fig. 4b). Its other component is opposed to the low-level wind, which is natural for a gravity wave drag. Where this force acts, $\mathcal{F}$ makes an angle with $\mathbf{U}\left(z_{w}\right)$ equal to $\pi / 2$ in the cold front case and equal to $-\pi / 2$ in the warm front case. From this, it follows that the horizontal component of the PV flux $\mathbf{J}_{N x y}$ is such that $\mathbf{J}_{N x y} \cdot \mathbf{U}\left(z_{w}\right)<0$ in the cold front case, whereas $\mathbf{J}_{N x y} \cdot \mathbf{U}\left(z_{w}\right)>0$ in the warm front case. Because of this difference, the surface response to the GWs in the warm front case (Figs. 8a-c) presents no symmetry with that in the cold front case (Figs. 5a-c). On the other hand, the response to the large-scale orography $\phi_{E}$ is the symmetric of the cold front response, in respect to the $x$ axis (Figs. $5 \mathrm{~d}-\mathrm{f}$ and $8 \mathrm{~d}-\mathrm{f}$ ).

In response to the PV pattern in Fig. 7, the surface response $\phi_{W}$ is dominated by a strong anticyclonic lobe, which stays attached to the mountain (Figs. 8a-c). Immediately downstream, according to $\mathbf{U}(0)$ a substantial trough develops. Note also that the magnitude of the response is stronger than in the cold front case (between 1.8 and $-1.2 \mathrm{mb}$ at $t=36 \mathrm{~h}$ ). It follows that the anticyclonic PV anomalies over the mountain and extending below $z_{w}$ downstream of it in Figs. 7b,c reinforce each other to produce a strong surface response near the mountain. By summing the two responses, it is apparent in Figs. $8 \mathrm{~g}-\mathrm{i}$ that in this case the absorbed GWs reinforce the anticyclone over the mountain, as well as the downslope trough.

\section{Sensitivity experiments}

\section{a. Quasigeostrophic simulations}

The fundamental reason for choosing the SG formalism rather than the QG formalism is that we obtain a more accurate PV budget, closer to the primitive equation PV budget. More precisely, in our case, the nonadvective PV flux $\mathbf{J}_{N}$ has a vertical component $J_{N z}$ that keeps $\mathbf{J}$ parallel to the tilted isentropes (Haynes and 

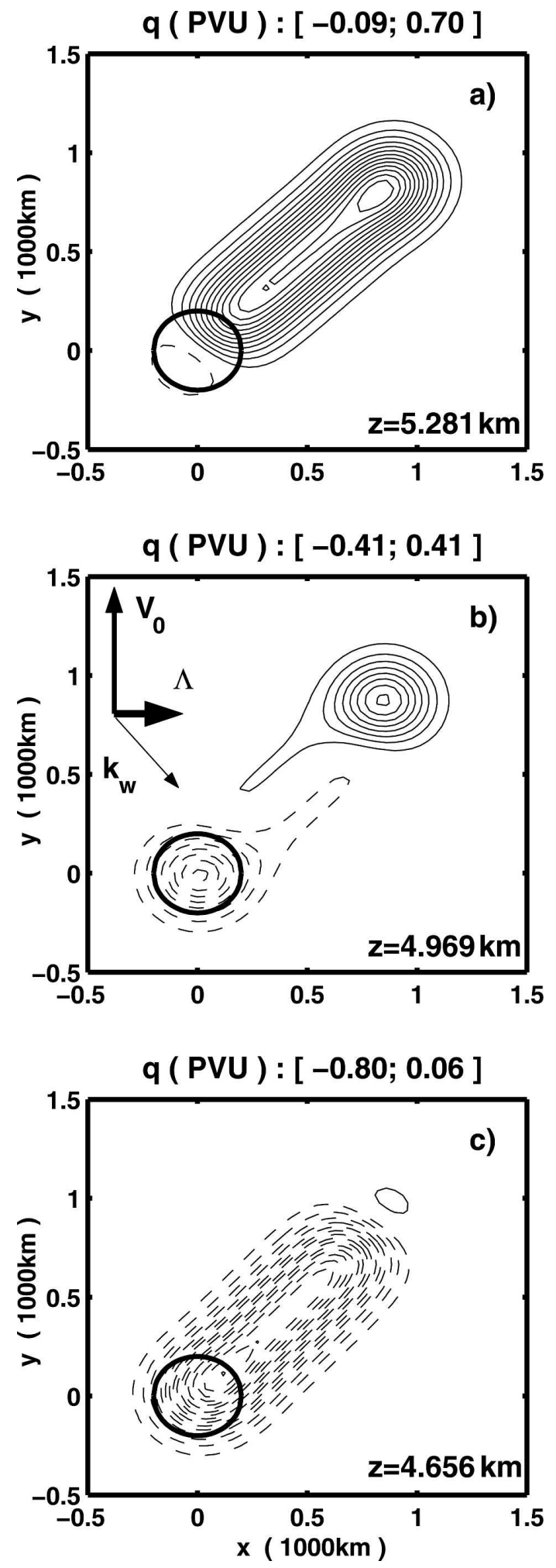

FIG. 7. Horizontal sections of the PV anomaly in the critical zone at $t=12 \mathrm{~h}$ and for different heights, for the warm front case in section 4: (a) $z=5.281 \mathrm{~km}$, (b) $z=4.969 \mathrm{~km}$, and (c) $z=4.656$ $\mathrm{km}$. Same parameters as in Fig. 2 except $V_{0}=20 \mathrm{~m} \mathrm{~s}^{-1}$ and $k_{w}=$ $-l_{w}$. Negative values are dashed and CI $=0.05$ PVU.
McIntyre 1987). This vertical component is neglected in the QG formalism. To evaluate its significance, we have repeated the cold and warm front experiments in sections 3 and 4 using a $Q G$ version of the model of section 2. To do it, we take $\Theta_{y} \mathcal{F}_{z}=\mathrm{Ri}^{-1}=0$ in the PV Eq. (12) and $\mathrm{Ri}^{-1}=\lambda_{i}=0$ in the definition of the vertical decay length $\lambda$ Eq. (14).

The most noticeable difference is that the warm front response is exactly antisymmetric with the cold front response according to the $x$ axis (Fig. 9). Because of the neglect of $J_{N z}$, at all altitudes the PV is due to a horizontal flux $\mathbf{J}_{N x y}$ that is aligned with the wind (not shown), so it resembles that at $z_{w}$ in the SG simulations (Figs. 3b and 7b). The QG simulations fail in simulating the substantial, sign-definite, and elongated PV anomaly located on the lower flank of the critical zone (Figs. 3c and 7c). That is the reason why the surface signal in the QG cases is much smaller than in the corresponding SG cases.

Nevertheless, we can notice that downstream of the mountain the GWs remain cyclolitic for a cold front case and cyclogenetic for a warm front. Over the mountain, the GWs still produce a low for the cold front case and a high for the warm front.

\section{b. Front, orography, and critical zone altitude}

The numerical simplicity of our model permits us to analyze many different flow and orography configurations. For example, increasing only $H_{0}$ favors the $\mathrm{GW}$ forcing [varying in $H_{0}^{2}$, Eq. (22)] relative to the largescale orography forcing [in $H_{0}$, Eq. (18)]. Increasing the number of ridges in our mountain (i.e., increasing $\left.\left|\mathbf{k}_{w}\right| L\right)$ results in the same effect, even if $L$ is fixed, and although the critical zone becomes thinner. Changing the orientation of the ridges [i.e., the angle $\left(\widehat{\mathbf{e}_{x}, \mathbf{k}_{w}}\right)$ ] has a more subtle effect. In the cold front case, for $0<$ $\left(\widehat{\mathbf{e}_{x}, \mathbf{k}_{w}}\right)<(\pi / 4)$, the GWs meet critical levels nearer the ground. It results in three effects: (i) the PV anomaly is nearer the ground, (ii) the advection of PV is slower, and (iii) the axis of the PV anomaly is closer to the surface wind $V_{0} \mathbf{e}_{y}$. As illustrated in Figs. 10a-c, this makes the surface response (i) thinner in width, (ii) shorter in length, and (iii) oriented closer to the surface wind $V_{0} \mathbf{e}_{y}$. The same results hold for the warm front case and for $-(\pi / 4)<\left(\widehat{\mathbf{e}_{x}, \mathbf{k}_{w}}\right)<0$ (not shown). The compromise of those different processes is summarized in Fig. 11, where the extrema of $\phi_{W}$ at $t=36 \mathrm{~h}$ are plotted versus $\left(\widehat{\mathbf{e}_{x}, \mathbf{k}_{w}}\right)$ in the cold and warm front cases. Notably, in both cases, the maximum amplitude is obtained for an intermediate value of $\left|\left(\widehat{\mathbf{e}_{x}, \mathbf{k}_{w}}\right)\right|$, and not for $\left|\left(\widehat{\mathbf{e}_{x}, \mathbf{k}_{w}}\right)\right| \approx 0$ for which $z_{w}$ is nearer the ground. The reason is that $z_{w} \approx 0$ requires $\mathbf{k}_{w} \cdot \mathbf{U}_{0} \approx 0$, that is, a 

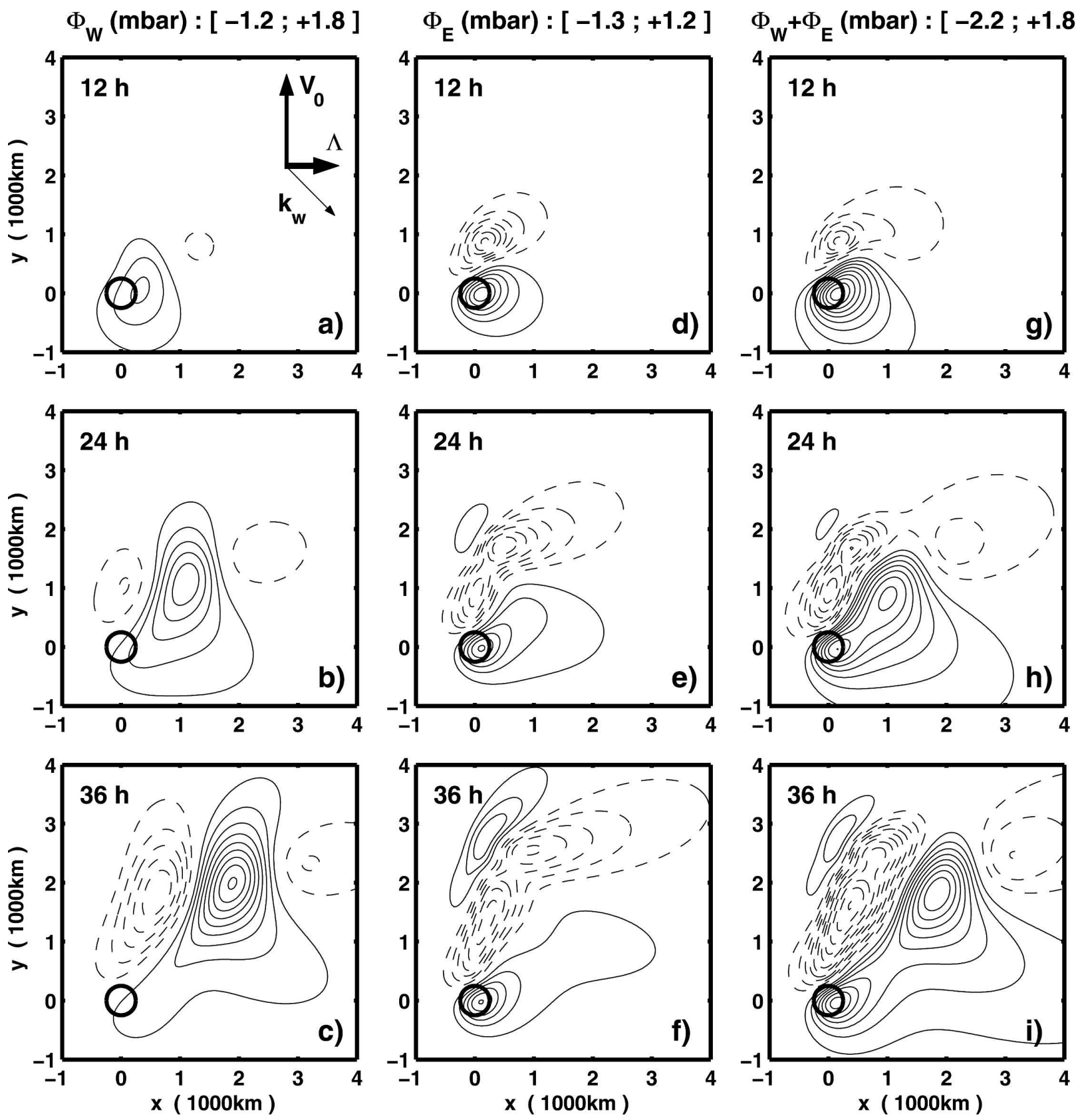

FIG. 8. Same as in Fig. 5 but in the warm front case (same parameters as in Fig. 7).

quasi-null cross-ridge velocity, and therefore a weak GW drag [see Eq. (A5) in the appendix]. The amplifying effect of the descent of the critical zone by decreasing $\left|\left(\widehat{\mathbf{e}_{x}, \mathbf{k}_{w}}\right)\right|$ is thus balanced by a decrease of the total force amplitude.

\section{c. Influence of the tropopause}

To study the impact of the geostrophic baroclinic instability on our results, we place a rigid lid at the alti- tude $D=10 \mathrm{~km}$. A conventional linear stability analysis for our model shows that unstable baroclinic modes have wavelengths exceeding $2 \pi /(2.6 \times f / N D) \sim 2400$ $\mathrm{km}$, and that the most unstable mode is oriented in the alongfront direction $\left(\Lambda=\Lambda \mathbf{e}_{x}\right)$ with a wavelength near $3700 \mathrm{~km}\left[\mathbf{k}=1.7 \times f /(N D) \mathbf{e}_{x}\right]$.

As shown in Figs. $12 \mathrm{a}-\mathrm{c}$ for the cold front case in section 3 , in the short term (12 h; Fig. 12a) the instability essentially has a slight quantitative effect on the surface pressure pattern, compared to the case with no 

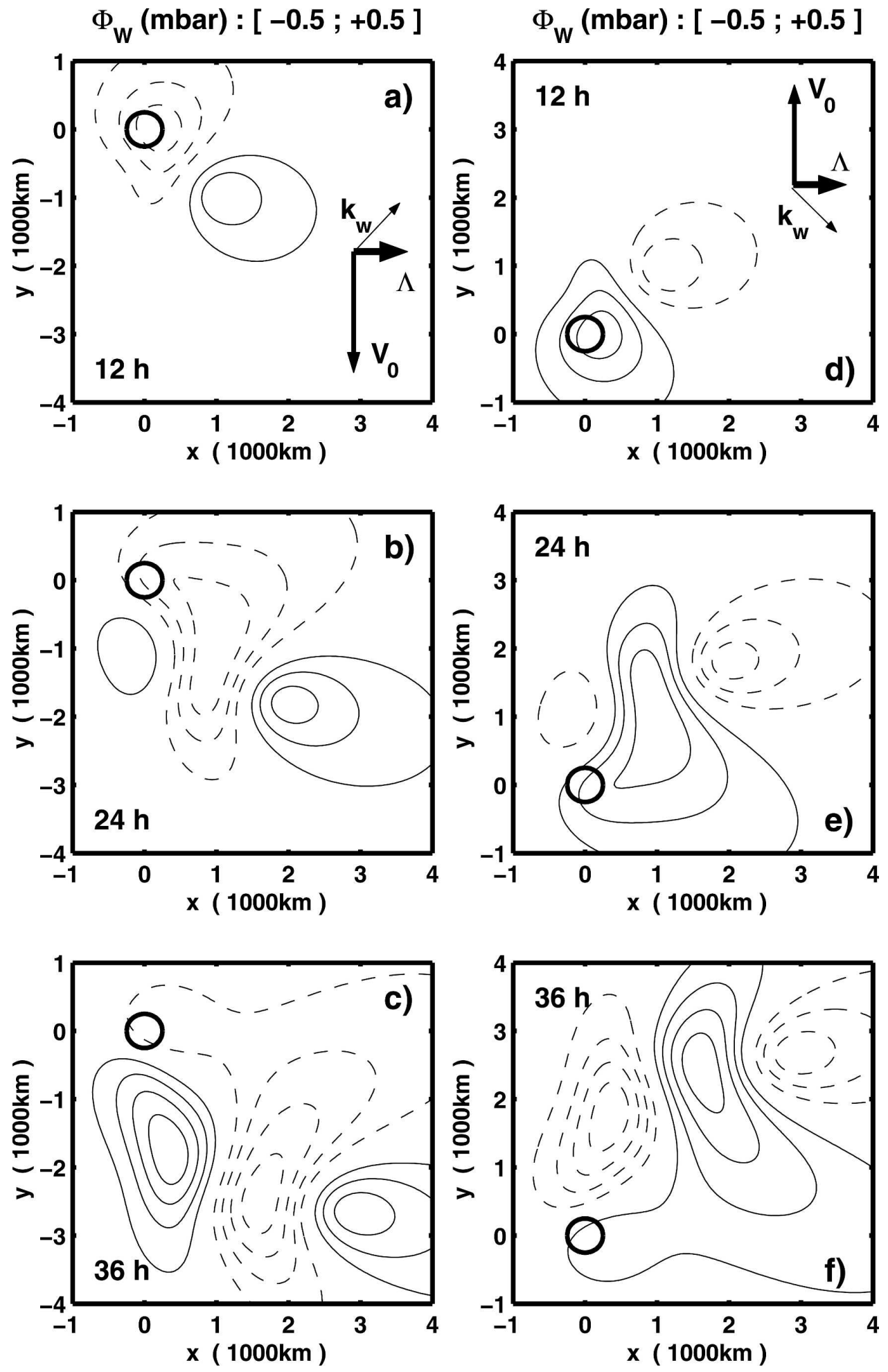

FIG. 9. Quasigeostrophic response to the GWs force. (a)-(c) Cold front; same parameters as in Fig. 5. (d)-(f) Warm front; same parameters as in Fig. 8. Negative values are dashed and CI $=0.1 \mathrm{mb}$. 

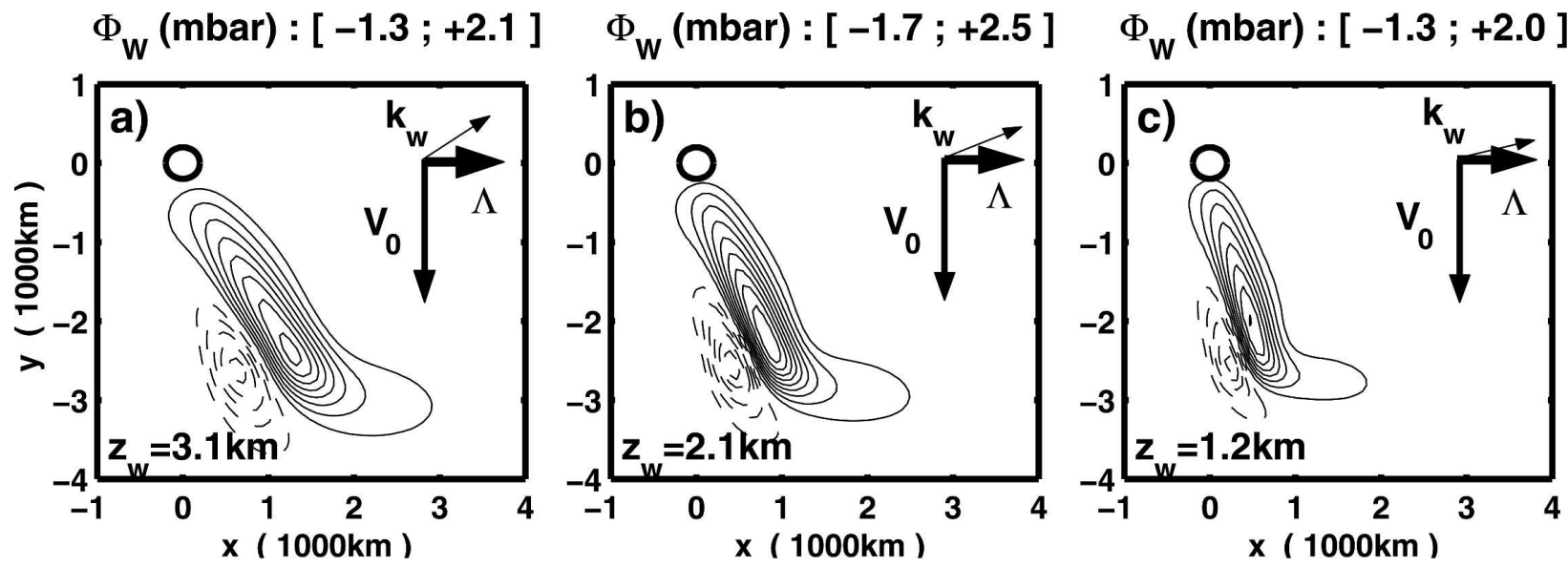

FIG. 10. Surface geopotential $\phi_{W}$ induced by the GWs at $t=36 \mathrm{~h}$ for three different orientations $\left(\widehat{\mathbf{e}_{x}, \mathbf{k}_{w}}\right)$, and for the cold front in section 3. Same background flow and orography parameters as in Fig. 2 except: (a) $\left(\widehat{\mathbf{e}_{x}, \mathbf{k}_{w}}\right)=0.7 \times \pi / 4$, (b) $\left(\widehat{\mathbf{e}_{x}, \mathbf{k}_{w}}\right)=0.5 \times \pi / 4$, and (c) $\left(\widehat{\mathbf{e}_{x}, \mathbf{k}_{w}}\right)=0.3 \times \pi / 4$. The corresponding altitude of the critical zone is also indicated; CI $=0.25 \mathrm{mb}$.

tropopause in Fig. 5a. At $t=24$ h (Fig. 12b), the presence of long unstable modes starts to modulate the response substantially. In particular, near and over the mountain the trough is more intense (see Fig. 5b), and its horizontal scale exceeds $2000 \mathrm{~km}$ along $\boldsymbol{\Lambda}$. Later $(t=$ 36 h; Fig. 12c), the long modes' influence is even stronger. Nevertheless, they do not much affect the maximum amplitude of the signal but rather spread it toward $\Lambda$. A qualitatively comparable behavior occurs in the warm front case of section 4 (Figs. 12d-f), except that it is quantitatively less pronounced.

\section{Extension to more realistic mountains}

The near monochromatic nature of the small-scale orography used until now results in a rather thin critical zone. In reality, however, mountains have broadband spectra and the GWs can interact with critical levels almost everywhere over the entire troposphere depth. To account for this, we follow Gregory et al. (1998) and represent the real orography spectrum over square domains of size $X \times Y$ by a spectral density function $A(\mathbf{k})$ that captures exactly the orographic variance a) COLD FRONT

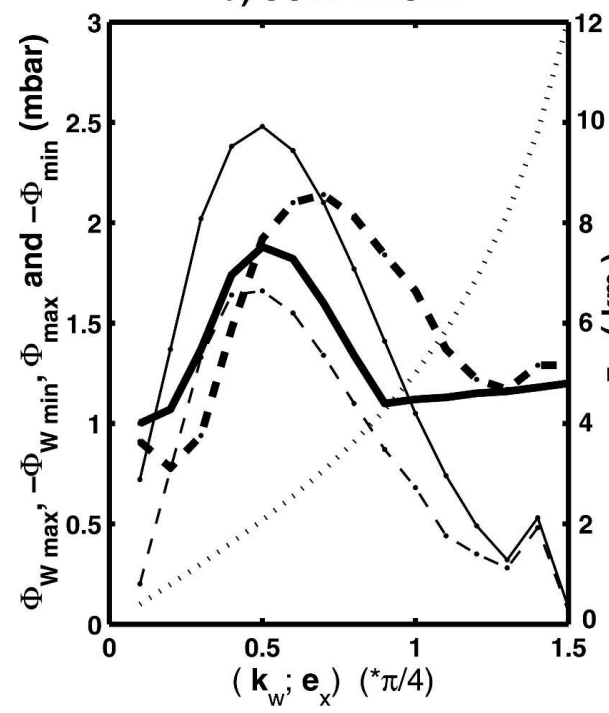

b) WARM FRONT

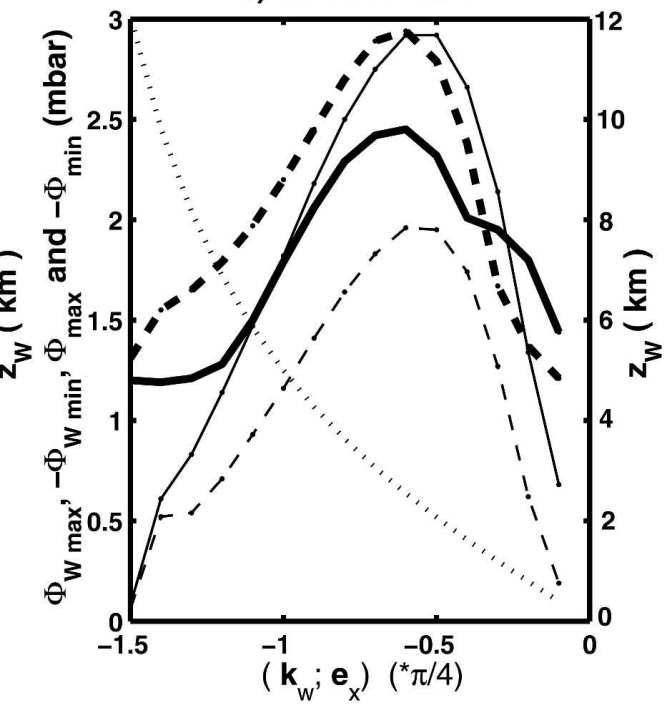

FIG. 11. Extrema in the surface geopotential due to the GWs at $t=36 \mathrm{~h}$ and for various angles $\left(\widehat{\mathbf{e}_{x}, \mathbf{k}_{w}}\right)$ : (a) cold front and (b) warm front. In both (a) and (b), $\max \left(\phi_{W}\right)$ is the thin solid line, $-\min \left(\phi_{W}\right)$ is thin dashed, $\max \left(\phi_{W}+\phi_{E}\right)$ is thick solid, $-\min \left(\phi_{W}+\phi_{E}\right)$ is thick dashed, and $z_{w}$ is dotted. 

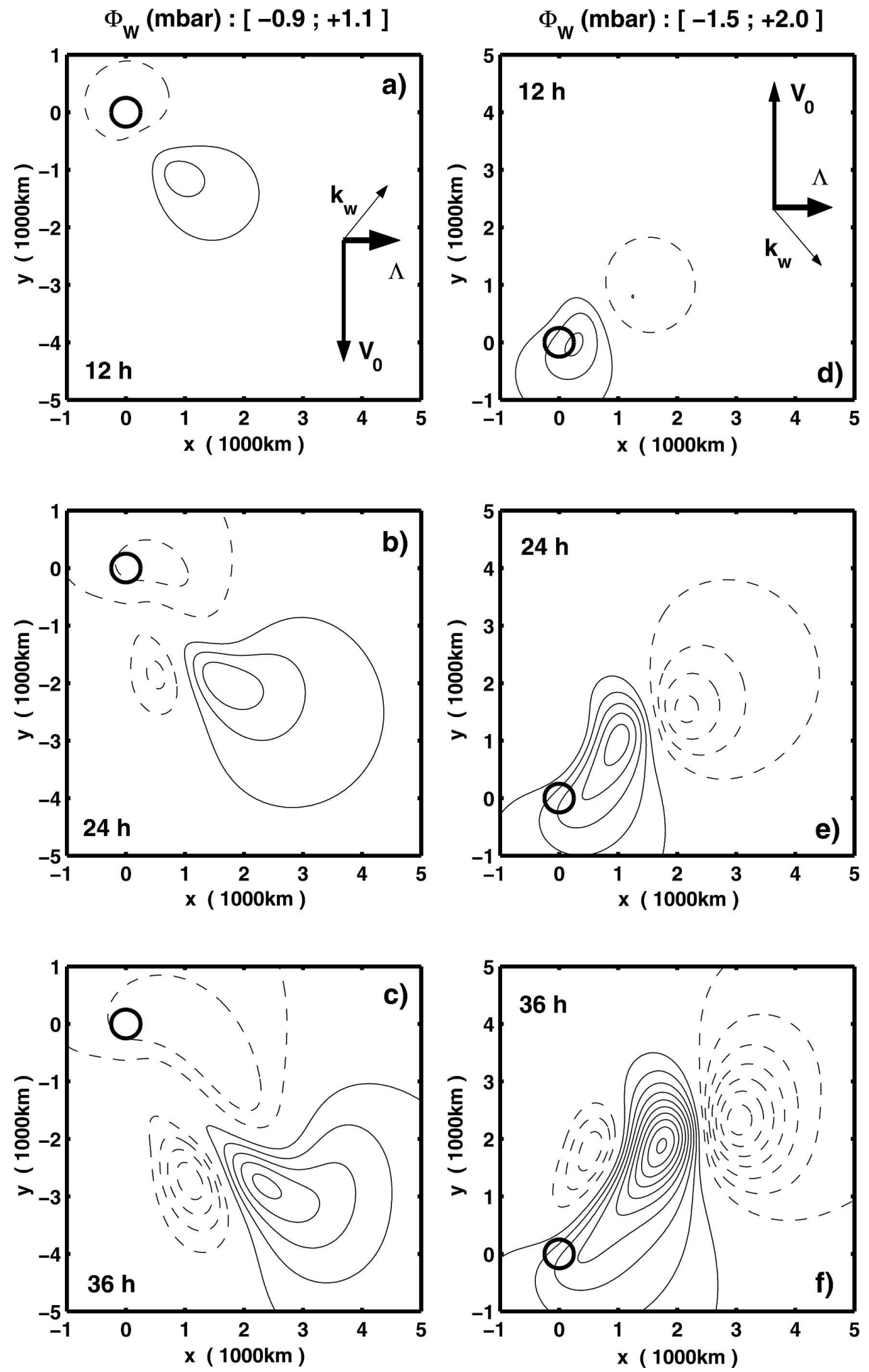

Fig. 12. Simulations with a tropopause at $D=10 \mathrm{~km}$. Cold front with the same parameters as in Fig. 5: (a) $t=12 \mathrm{~h}$, (b) $t=24 \mathrm{~h}$, and (c) $t=36 \mathrm{~h}$. Warm front with the same parameters as in Fig. 8: (d) $t=12 \mathrm{~h}$, (e) $t=24 \mathrm{~h}$, and (f) $t=36 \mathrm{~h}$. Negative values are dashed and CI $=0.2 \mathrm{mb}$. 


$$
\begin{aligned}
\frac{1}{X Y} \int_{0}^{X} \int_{0}^{Y} h^{\prime 2} d x d y & =\int_{0}^{+\infty} \int_{0}^{2 \pi} \frac{4 \pi^{2}}{X Y}|\hat{h}(K, \alpha)|^{2} K d K d \alpha \\
& =\int_{0}^{+\infty} \int_{0}^{2 \pi} A(K, \alpha) K d K d \alpha .
\end{aligned}
$$

In Eq. (33) $K$ and $\alpha$ are the polar coordinates of $\mathbf{k}$, and $\hat{h}(K, \alpha)$ is the Fourier transform of $h^{\prime}$ inside of the domain $X \times Y$, which can be estimated for real data by the FFT coefficient $\tilde{h}$ calculated from the discrete series of $h^{\prime}$. We then assume an isotropic spectral density in the wavenumber band relevant for gravity waves

$$
K A(\mathbf{k})=a\left(\frac{K_{0}}{K}\right)^{\gamma}, \text { for } \quad K_{L}<\|\mathbf{k}\|<K_{U}
$$

In Eq. (34) $K_{0}$ and $\gamma$ are constants that vary regionally, and $a=1 \mathrm{~m}^{3}$ is introduced for dimension consistency. We take $K_{U}=2 \pi /(10 \mathrm{~km}) \approx N / V_{0}$ because disturbances with shorter wavelength do not propagate vertically, and $K_{L}=2 \pi / \sqrt{X^{2}+Y^{2}}$, that is, approximately the longest harmonic the domain $X \times Y$ can represent. Note that we could have defined $K_{L}$ as the lower cutoff of the stationary inertia GWs, $K_{L}=f / V_{0} \approx 1200 \mathrm{~km}$. This is larger than the size of our domain, and hence is not represented in our spectrum analysis. Nevertheless, we have checked that the following analyses are not sensitive to $K_{L}$; for such low values of $\gamma$, the orographic variance concentrates on the small scales.

To evaluate the effect of this spectrum in the context of our model, we consider the effect of the small-scale mountains located in a domain of size $\pi L^{2}=X Y$ and use the method in the appendix to evaluate the net force due to $A(\mathbf{k})$ :

$$
\begin{aligned}
\overline{\mathcal{F}}= & \frac{2 N \Lambda a}{2-\gamma}\left[\left(\frac{K_{0}}{K_{U}}\right)^{\gamma} K_{U}^{2}-\left(\frac{K_{0}}{K_{L}}\right)^{\gamma} K_{L}^{2}\right] \frac{\Lambda z\left|V_{0}\right|^{3}}{\|\mathbf{U}(z)\|^{4}} \\
& \times\left[\mathbf{e}_{x}-\frac{U(z)}{V_{0}} \mathbf{e}_{y}\right] .
\end{aligned}
$$

We then distribute this force regionally as in section 2 , $\mathcal{F}(\mathbf{x}, z)=\overline{\mathcal{F}}(z) e^{-\left(x^{2}+y^{2} / L^{2}\right)}$.

To give realistic values to the parameters $K_{0}$ and $\gamma$, we have considered the Alpine sector $\left(43^{\circ}-48^{\circ} \mathrm{N}, 5^{\circ}-\right.$ $16^{\circ} \mathrm{E}$ ) and used the 2-Minute Gridded Global Relief Data (ETOPO2; around 2-km horizontal resolution; see the National Geophysical Data Center 2006). In this sector, we define 30 partially overlapping square domains, each of size $X Y=352 \mathrm{~km}^{2}=\pi L^{2}$, giving $L=$ $200 \mathrm{~km}$ as in sections 3, 4, and 5 (Fig. 13a). For each of these domains, we estimate $\left[\gamma, \ln \left(K_{0}^{\gamma}\right)\right]$ by a best square fit between $A(\mathbf{k})$ and the azimuthal mean of the orography periodogram $\tilde{h} \tilde{h}^{*}$. An example for one particular box $\left(43^{\circ} 48^{\prime} \mathrm{N}, 46^{\circ} 58^{\prime} \mathrm{N}\right) \times\left(5^{\circ} 26^{\prime} \mathrm{E}, 9^{\circ} 58^{\prime} \mathrm{E}\right)$ is shown in Fig. 13. For this box, the best fit is $\gamma=0.96$ and $\ln \left(K_{0}^{\gamma}\right)=10.2$ (Fig. 13b). For other boxes, the results are summarized in Fig. 13c; even if the parameters $\gamma$ and $\ln \left(K_{0}^{\gamma}\right)$ have significant variations (the range obtained for $\gamma$ is from 0.56 to 1.02), the resulting force for each box has a rather stable magnitude around $2 \times$ $10^{-4} \mathrm{~kg} \mathrm{~m}^{-2} \mathrm{~s}^{-2}$. Therefore, in our simulations we can use, for the force, the mean values

$$
\gamma=0.76 ; \quad \ln \left(K_{0}^{\gamma}\right)=13.5
$$

Note that our $\gamma$ is smaller than in Gregory et al. (1998; who found $\gamma>1$ ), probably because the Alps are sharper than the mountains studied by these authors.

Figures $14 \mathrm{a}, \mathrm{b}$ present the vertical profiles of $\mathcal{F}$ obtained with Eqs. (35)-(36) and for the cold front of section 3. As expected, the critical zone is deeper than in Fig. 2 and covers the entire troposphere. The surface response to this force in the absence of tropopause is shown in Fig. 14c at $t=36 \mathrm{~h}$. It resembles very much that of the idealized case in Fig. 5c. A predominant ridge is followed by a weaker trough, both being embedded in the south-southeast sector. The dominant wave vector is very similar to that of section $3 \mathrm{~b}$, although not so well defined. The reason is that the critical zone is deep, giving no precise horizontal axis to the PV pattern.

\section{Discussion and conclusions}

This paper gives a heuristic evaluation of the significance of mountain GWs for the synoptic-scale dynamics near large mountain ranges, in the presence of directional critical levels. The model used is an extension of the model of Smith (1984) for lee cyclogenesis. It includes a representation of the GWs adapted from Shutts (1995). The interaction between the GWs and the large-scale flow occurs via the PV anomaly, which is produced by the force due to the GWs, which encounter critical levels in the troposphere.

Section 3 details this interaction for a cold front, in the case where there is a good separation between the small-scale dynamics and the large-scale dynamics. In a configuration such that almost all the GWs encounter critical levels, they induce a force in a thin critical zone centered around a well-defined altitude $z_{w}$. The level $z_{w}$ depends on the configuration of the front relative to the orientation of the anisotropic small-scale orography. This force is everywhere perpendicular to the background flow and produces a PV anomaly around $z_{w}$ that is advected and steered by the background wind. 

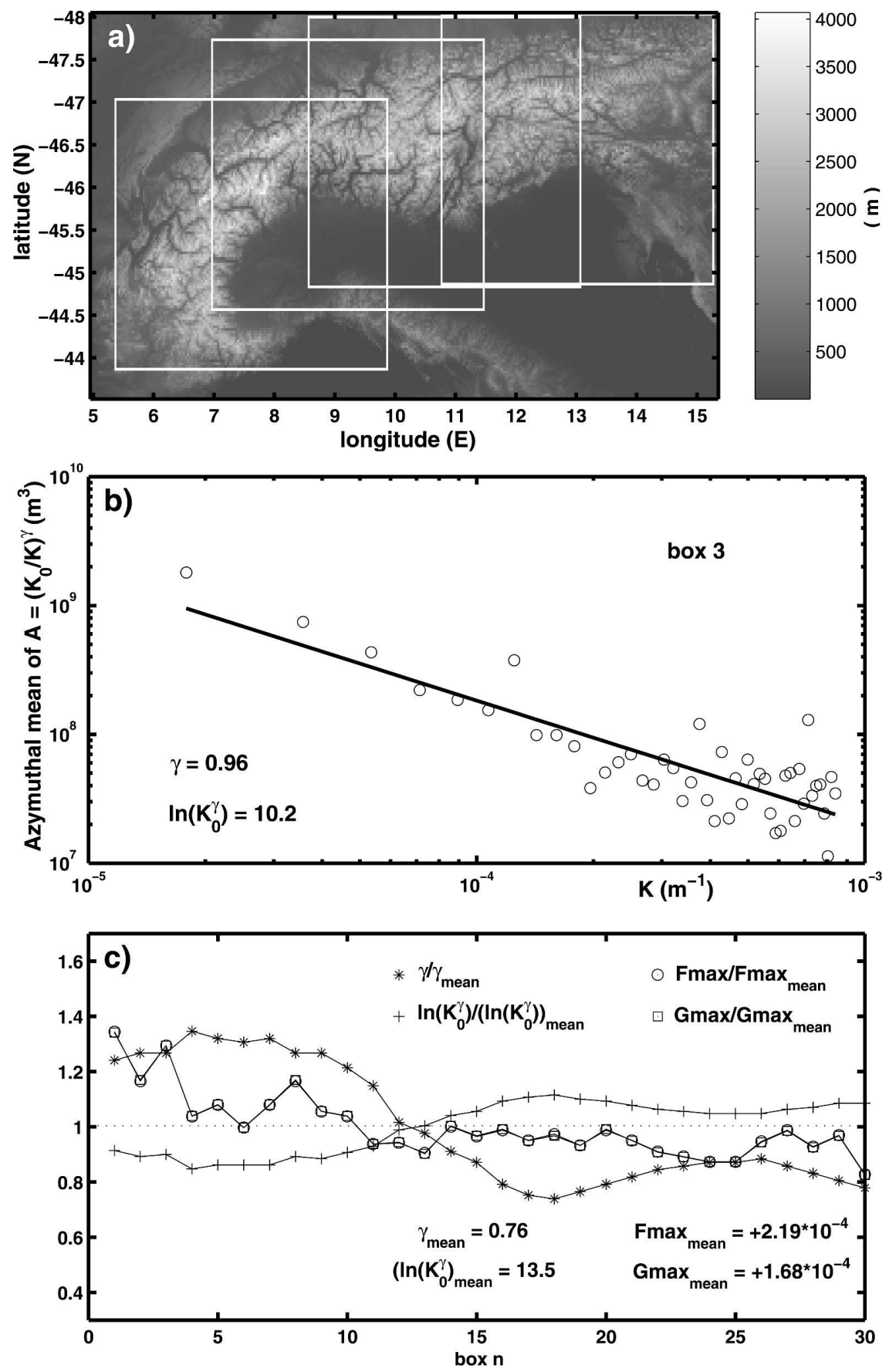

FIG. 13. Spectral analysis of the Alps. (a) Elevation from ETOPO2. Five among the 30 boxes used to evaluate the mean spectrum are indicated for illustration. (b) Azimuthal mean of the periodigram (circles) and best square linear fit (thick solid) for box 3 shown in the lower left of (a). (c) Best fit values for the 30 boxes: $\gamma$ (solid and star), $\ln \left(K_{0}^{\gamma}\right)$ (solid and plus), $\mathcal{F}_{\text {max }}$ (solid and circles), and $\mathcal{G}_{\max }$ (solid and square). All values in (c) are divided by their mean value over the 30 boxes.

Near $z_{w}$, the PV anomaly is predominantly cyclonic aloft of the mountain, and it presents a start-up anticyclone that travels at the velocity $\mathbf{U}\left(z_{w}\right)$ away from the mountain (Fig. 3). Below $z_{w}$ the PV anomaly is pre- dominantly anticyclonic, whereas above $z_{w}$ it is predominantly cyclonic. Accordingly, the surface response at small times (12 h in Fig. 5a) presents a trough over the mountain and a ridge downstream. Over a longer 

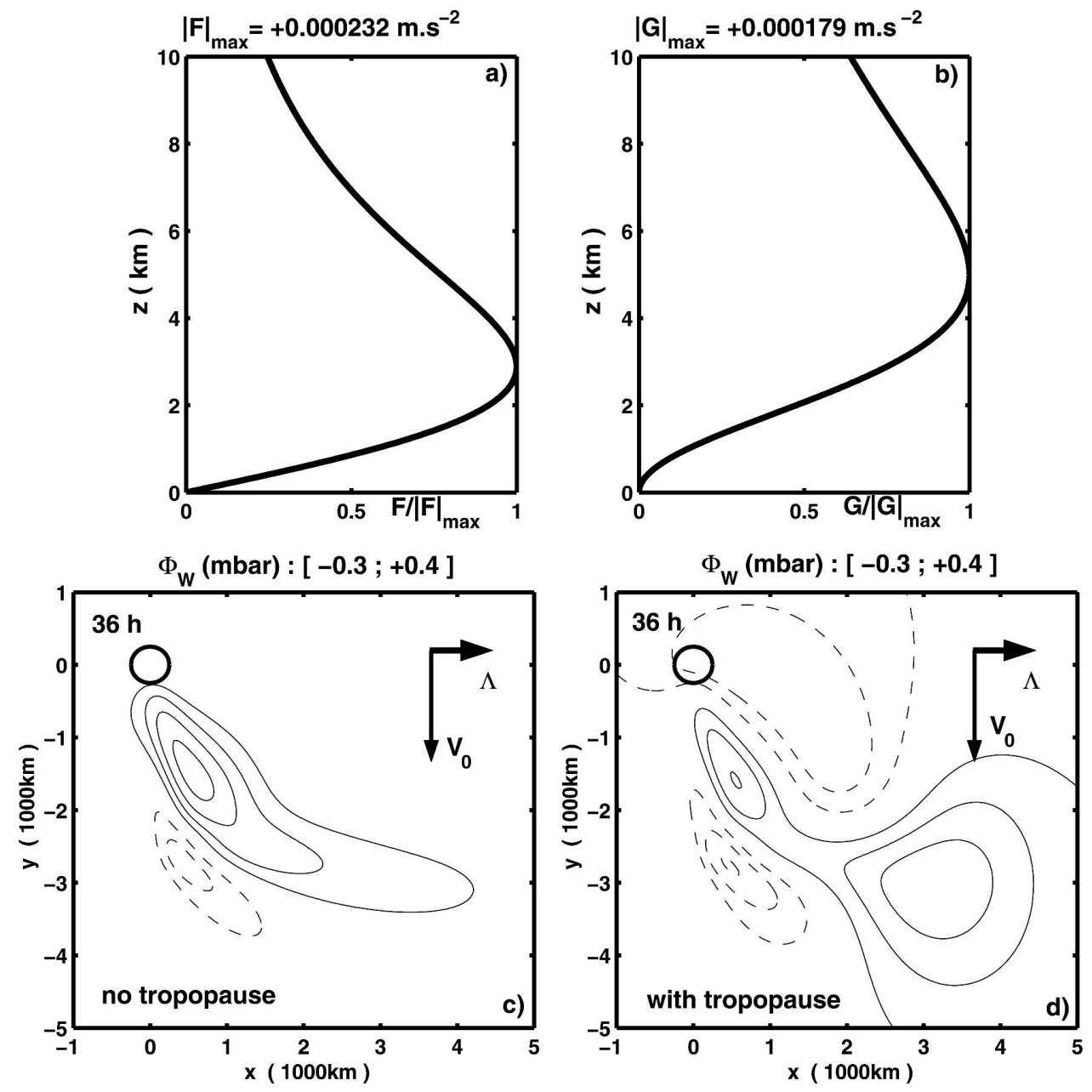

FIG. 14. Cold front simulations with an orographic spectrum representative of the Alps. (a) Vertical profile of $\overline{\mathcal{F}}(z)$, (b) vertical profile of $\overline{\mathcal{G}}(z)$, (c) $\phi_{W}$ at $t=36 \mathrm{~h}$ with no tropopause, and (d) $\phi_{W}$ at $t=36$ h with tropopause at $D=10 \mathrm{~km}$. Negative values are dashed and CI $=0.1 \mathrm{mb}$.

term, this response is modulated by Eady waves (Figs. $5 \mathrm{~b}, \mathrm{c})$; the trough over the mountain becomes quite small, and an elongated ridge builds up immediately downstream toward $\mathbf{U}\left(z_{w}\right)$. The GWs thus tend to slightly reduce the amplitude of the response to the large-scale orography, notably the ridge over the mountain and the lee trough (Figs. 5d-f). As the lee trough is associated with lee-cyclone initiation in Smith (1984), we can argue that the GWs are cyclolytic in the cold front case.

In section 4 we repeat the same study in the warm front case (Figs. 7 and 8), after rotating by $90^{\circ}$ the orientation of the mountain ridges in order that the GWs exert a substantial force. In this case, the PV anomaly produced by the GWs is predominantly anticyclonic over the mountain and cyclonic downstream (Fig. 7). It is also predominantly anticyclonic below $z_{w}$ and cyclonic above. Hence, the surface response presents a large-scale ridge attached to the mountain (Figs. 8a-c), and extending below the PV anomaly, toward $\mathbf{U}\left(z_{w}\right)$ (Fig. 7). Eady wave dynamics produce a trough downstream of the mountain, toward the surface wind $\mathbf{U}(0)$ (Figs. 8b,c). In this case, and contrary to the cold front case, the GWs reinforce the ridge over the mountain and the downstream trough (Figs. 8d-f).

The rest of the paper consists of doing tests of the robustness of these results. They indeed seem to be linked to geometrical properties of the GW directional critical levels, indicating that they are rather systematic. In section 5a, we adopt the QG framework. This illustrates the important role of the vertical component of the PV flux in baroclinic environments. In section 5b, we change the orientation of $\mathbf{k}_{w}$, and other crucial parameters. Even if those changes have some quantitative effects on the amplitude or the horizontal scale of the surface response, the cyclolytic nature of the GWs in 
the cold front case stays unchanged. The same is true in the warm front case, where the interaction remains cyclogenetic. The introduction of a tropopause in section $5 \mathrm{c}$ is more significant qualitatively, even if in our model, the baroclinic instabilities can only modulate the GW response at horizontal scales much larger than discussed up to now. The effect is particularly pronounced in the cold front case (Figs. 12a-c), where, compared to the same case without a tropopause (Figs. 5a-c), the trough over the ridge becomes rather large in the long term (Fig. 12c) instead of becoming small (Fig. 5c), while the ridge downstream becomes rather small instead of being large. In the warm front case (not shown) the effect of the GWs aloft of the mountain and immediately downstream of it stays the same as that without a tropopause. It is only in the far field and at large scales that the influence of the baroclinic instabilities becomes substantial.

In section 6 we present an extension of our model to more realistic small-scale orographic spectra. We use for this a method presented in Gregory et al. (1998) in the context of the parameterization of mountain GWs via a spectral method in the Met Office (UKMO) model. This method consists again of evaluating the GW force exactly but using a realistic spectral density function for the orography. For the case of the Alps (Fig. 13), the GW force is more broadly distributed over the troposphere. Nevertheless, the surface responses to the GWs (Fig. 14) share many properties with the responses for the idealized orography profiles used in sections 3,4 , and 5 .

Despite the attractive simplicity of our model, these results should be interpreted with some caution. For instance, the neglect of unbalanced effect can be inaccurate for shears larger than here; the inertio-gravity waves generated during the interaction (Lott 2003; Vadas et al. 2003; Shutts 2003) or the nongeostrophic unstable baroclinic modes (Plougonven et al. 2005) may have a substantial influence at the surface. More seriously, by representing the fronts by uniform shears we do not consider the barotropic growth of unstable modes that the smooth mountain and the GWs may trigger. This may result in effects rather different from those discussed here, in particular because the horizontal scale of the unstable modes may be much closer to the horizontal scale of the mountain.

Nevertheless, we believe that the structure of the PV anomalies we discuss here is of some interest. They are produced by PV fluxes that are everywhere parallel to the background wind, a situation rarely discussed in the literature. As a result, they do not resemble the horizontal PV banners that are often discussed. These PV anomalies are also strongly affected by the inclination of the isentropes. For these reasons, they may reflect some aspects of the mesoscale and synoptic-scale PV patterns found in the observations. For example, Hoinka et al. (2003) have carried out an analysis of the MAP intensive observation period 15 (IOP-15) in which a PV streamer from the low stratosphere passes above the Alps, triggering lee cyclogenesis. They mention the importance of the breaking GWs in the cutoff process and the presence of enhanced shear. Since the order of magnitude of our PV anomalies is comparable to theirs, the process we have studied here might have some relevance in their case.

Another interest of this study is to give a theoretical approach to the problem of mountain GW parameterization in large-scale models. In this respect, the fact that the response to the GWs is generally smaller than the response to the large-scale orography is reasonable. The SSO parameterizations are intended for correcting errors, not for producing the dominant signal. In this context, it gives two messages.

First, a rather systematic error of large-scale models is that they underestimate, at least in winter, the anticyclonic circulation that occurs over the large-scale mountains. These errors have been corrected in the past either by introducing an envelope orography, or by introducing lift forces oriented perpendicularly to the geostrophic flow and toward the right (in the Northern Hemisphere). The two approaches are in good part equivalent (Lott 1999). Although the GW force is also perpendicular to the flow, our results show that it does not systematically help the building up of anticyclones over mountains. Indeed, it is probably the other way around because cold fronts are stronger than warm fronts in a climatological sense. Second, our results show that absorbed GWs have little chance to help lee cyclogenesis and seem to have a rather cyclolytic influence, if we again assume that cold fronts are more frequent.

\section{APPENDIX}

\section{Evaluation of the GW Force $\overline{\mathcal{F}}$}

We use the linear Boussinesq theory of hydrostatic stationary mountain GWs in uniform background shears (Shutts 1995). The vertical velocity for each monochromatic wave satisfies

$$
\begin{aligned}
\frac{\partial^{2} \hat{w}^{\prime}}{\partial z^{2}}+\frac{N^{2}}{[\mathbf{k} \cdot \mathbf{U}(z)]^{2}}\left(k^{2}+l^{2}\right) \hat{w}^{\prime} & =0, \quad \text { with } \\
\hat{w}^{\prime}(0) & =-i \mathbf{k} \mathbf{U}_{0} \hat{h}^{\prime} .
\end{aligned}
$$


The waves that present a critical level at $z_{c}=-\mathbf{k} \mathbf{U}_{0} / k \Lambda>$ 0 can be written

$$
\hat{w}^{\prime}(z)=-i \mathbf{k} \mathbf{U}_{0} \hat{h}^{\prime}\left(1-\frac{z}{z_{c}}\right)^{1 / 2+\epsilon i \alpha}, \quad \epsilon= \pm 1, \text { for } z<z_{c}
$$

$$
\approx 0 \quad \text { for } \quad z>z_{c}
$$

where $\alpha=\sqrt{\operatorname{Ri}\left(k^{2}+l^{2} / k^{2}\right)-1 / 4}$. We take $\epsilon \mathbf{k} \mathbf{U}_{0}>0$ to impose an upward group velocity. In Eqs. (A2)-(A3) the GWs reflected or transmitted at $z_{c}$ have been neglected because $\mathrm{Ri} \approx 6 \gg 0.25$ (Booker and Bretherton 1967). Note that Eq. (A2) is an exact solution of Eq. (A1) for the entire domain under $z_{c}$. Besides, the mean stress satisfies

$$
\begin{aligned}
\overline{\mathbf{u}^{\prime} w^{\prime}} & \equiv \frac{1}{\pi L^{2}} \int_{-\infty}^{+\infty} \int_{-\infty}^{+\infty} \mathbf{u}^{\prime} w^{\prime} d x d y \\
& =\frac{4 \pi^{2}}{\pi L^{2}} \int_{k=0}^{+\infty} \int_{l=-\infty}^{+\infty}\left(\hat{\mathbf{u}} \hat{w}^{*}+\hat{\mathbf{u}}^{*} \hat{w}\right) d l d k .
\end{aligned}
$$

We take the background wind of Eq. (3) for a cold front $\left(V_{0}<0, \Lambda>0\right)$, and use Eqs. (A2)-(A3) and the dispersion relationships for the GWs, which yields

$$
\begin{aligned}
\overline{\mathbf{u}^{\prime} w^{\prime}}(z)= & \frac{8 \pi^{2}}{\pi L^{2}} \int_{k=0}^{+\infty} \int_{l=-\frac{k U(z)}{\mathrm{v}_{0}}}^{+\infty}-\frac{\mathbf{k}}{\|\mathbf{k}\|}\left(\mathbf{k} \mathbf{U}_{0}\right) N\left|\hat{h}^{\prime}\right|^{2} d l d k \\
& +\ldots,
\end{aligned}
$$

where the dots are for the $z$-independent part of the flux carried by the GWs that do not encounter critical levels. Then, the associated force is

$$
\begin{aligned}
\overline{\mathcal{F}}(z)= & -\frac{d}{d z} \overline{\mathbf{u}^{\prime} w^{\prime}}=\frac{8 \pi^{2}}{\pi L^{2}} N \Lambda \\
& \times \int_{0}^{+\infty} k^{2}\left|\hat{h}^{\prime}\left[k, l=-k \frac{U(z)}{V_{0}}\right]\right|^{2} d k \\
& \times \frac{\Lambda z}{\|\mathbf{U}(z)\|}\left[\mathbf{e}_{x}-\frac{U(z)}{V_{0}} \mathbf{e}_{y}\right] .
\end{aligned}
$$

This last formula is valid for any kind of front of the form of Eq. (3), cold or warm. For the idealized orography profile of Eq. (1), $h^{\prime}=\mathcal{H}(\mathbf{x}) \cos \left(\mathbf{k}_{w} \mathbf{x}\right)$, which leads to

$$
\hat{h}^{\prime}=\frac{H_{0} L^{2}}{4 \pi}\left(e^{-\left(\mathbf{k}-\mathbf{k}_{w}\right)^{2}\left(L^{2} / 2\right)}+e^{-\left(\mathbf{k}+\mathbf{k}_{w}\right)^{2}\left(L^{2} / 2\right)}\right) .
$$

To simplify the analytical treatment under the assumption that the horizontal scales are clearly separable
( $\left\|\mathbf{k}_{w}\right\| L \gg 1$ ), we neglect the second Gaussian term of Eq. (A7), because it is centered around $-\mathbf{k}_{w}$ and the integral in Eq. (A6) is for $k>0$. The steepest descent estimate of the integral of the remaining Gaussian term in Eq. (A6) yields the Eqs. (22)-(23) in section 2c. Note that if $k_{w} l_{w}<0$ for the cold front case, we obtain $\overline{\mathcal{F}} \approx$ 0 , because the waves that encounter a critical level have a very small amplitude in this case. On the opposite, in the warm front case, $\overline{\mathcal{F}} \approx 0$ if $k_{w} l_{w}>0$.

\section{REFERENCES}

Aebischer, U., and C. Schär, 1998: Low-level potential vorticity and cyclogenesis to the lee of the Alps. J. Atmos. Sci., 55, 186-207.

Binder, P., and C. Schär, 1996: MAP design proprosal. Meteo Swiss, 77 pp. [Available online at http://www.map.meteoswiss. ch/map-doc/prop/MDP.pdf.]

Boer, G. J., N. A. McFarlane, R. Laprise, J. D. Henderson, and J.-P. Blanchet, 1984: The Canadian Climate Center spectral atmospheric general circulation model. Atmos.-Ocean, 22, 397-429.

Booker, J. R., and F. P. Bretherton, 1967: The critical layer for internal gravity waves in a shear flow. J. Fluid Mech., 27, 513-539.

Bougeault, P., and Coauthors, 2001: The MAP special observing period. Bull. Amer. Meteor. Soc., 82, 433-462.

Bretherton, F. P., 1969: Momentum transport by gravity waves. Quart. J. Roy. Meteor. Soc., 95, 213-243.

Broad, A. S., 1999: Do orographic gravity waves break in flows with uniform wind direction turning with height? Quart. J. Roy. Meteor. Soc., 125, 1695-1714.

Bühler, O., and M. E. McIntyre, 2005: Wave capture and wavevortex duality. J. Fluid Mech., 534, 67-95.

Davis, C. A., 1997: The modification of baroclinic waves by the Rocky Mountains. J. Atmos. Sci., 54, 848-868.

Eliassen, A., and E. Palm, 1961: On the transfer of energy in the stationary mountain waves. Geofys. Publ., 22, 1-23.

Fantini, M., and S. Davolio, 2001: Instability of neutral Eady waves and orography. J. Atmos. Sci., 58, 1146-1154.

Flamant, C., E. Richard, C. Schär, R. Rotunno, L. Nance, M. Sprenger, and R. Benoit, 2004: The wake south of the Alps: Dynamics and structure of the lee-side flow and secondary potential vorticity banners. Quart. J. Roy. Meteor. Soc., 130, $1275-1303$.

Gregory, D., G. J. Shutts, and J. R. Mitchell, 1998: A new gravitywave-drag scheme incorporating anisotropic orography and low-level wave-breaking: Impact upon the climate of the UK Meteorological Office unified model. Quart. J. Roy. Meteor. Soc., 124, 463-493.

Gross, B. D., 1994: Frontal interaction with isolated orography. $J$. Atmos. Sci., 51, 1480-1496.

Haynes, P. H., and M. E. McIntyre, 1987: On the evolution of vorticity and potential vorticity in the presence of diabatic heating and frictional or other forces. J. Atmos. Sci., 44, 828841.

Hoinka, K. P., E. Richard, G. Poberaj, R. Busen, J.-L. Caccia, A. Fix, and H. Mannstein, 2003: Analysis of a potential-vorticity streamer crossing the Alps during MAP IOP-15 on 6 November 1999. Quart. J. Roy. Meteor. Soc., 129, 609-632.

Hoskins, B. J., 1975: The geostrophic momentum approximation 
and the semi-geostrophic equations. J. Atmos. Sci., 32, 233242.

— M. E. McIntyre, and A. W. Robertson, 1985: On the use and significance of isentropic potential vorticity maps. Quart. J. Roy. Meteor. Soc., 111, 877-946.

Jiang, Q., and J. D. Doyle, 2004: Gravity wave breaking over the central Alps: Role of complex terrain. J. Atmos. Sci., 61, 2249-2266.

Liniger, M. A., and H. C. Davies, 2003: Substructure of a map streamer. Quart. J. Roy. Meteor. Soc., 129, 633-651.

Lott, F., 1999: Alleviation of stationary biases in a GCM through a mountain drag parameterization scheme and a simple representation of mountain lift forces. Mon. Wea. Rev., 127, 788801.

_ 2003: Large scale flow response to short gravity waves breaking in a rotating shear flow. J. Atmos. Sci., 60, 16911704.

— rametrization: Its formulation and testing. Quart. J. Roy. Meteor. Soc., 123, 101-127.

McFarlane, N. A., 1987: The effect of orographically excited gravity wave drag on the general circulation of the lower stratosphere and troposphere. J. Atmos. Sci., 44, 1775-1800.

Messinger, M., and R. Pierrehumbert, 1986: Alpine lee cyclogenesis: Numerical simulation and theory. Scientific Results of the Alpine Experiment, GARP Publications Series 27, Vol. I, WMO/International Council of Scientific Unions, 141-165.

Miller, M. J., T. N. Palmer, and R. Swinbank, 1989: Parametrization and influence of subgridscale orography in general circulation and numerical weather prediction models. Meteor. Atmos. Phys., 40, 84-109.

National Geophysical Data Center, cited 2006: 2-Minute Gridded Global Relief Data (ETOPO2v2). NOAA/NESDIS/NGCD. [Available online at http://www.ngdc.noaa.gov/mgg/fliers/ 06mgg01.html.]

Palmer, T. N., G. J. Shutts, and R. Swinbank, 1986: Alleviation of a systematic westerly bias in general circulation and numerical weather prediction models through an orographic gravity wave drag parametrization. Quart. J. Roy. Meteor. Soc., 112, 1001-1039.

Phillips, D. S., 1984: Analytical surface pressure and drag for linear hydrostatic flow over three-dimensional elliptical mountains. J. Atmos. Sci., 41, 1073-1084.

Pierrehumbert, R. T., 1985: A theoretical model of orographically modified cyclogenesis. J. Atmos. Sci., 42, 1244-1258.
Plougonven, R., D. J. Muraki, and C. Snyder, 2005: A baroclinic instability that couples balanced motions and gravity waves. J. Atmos. Sci., 62, 1545-1559.

Scavuzzo, C. M., M. A. Lamfri, H. Teitelbaum, and F. Lott, 1998: A study of the low frequency inertio-gravity waves observed during PYREX. J. Geophys. Res., 103 (D2), 1747-1758.

Schär, C., 1990: Quasi-geostrophic lee cyclogenesis. J. Atmos. Sci., 47, 3044-3066.

- and D. R. Durran, 1997: Vortex formation and vortex shedding in continuously stratified flows past isolated topography. J. Atmos. Sci., 54, 533-554

- M. Sprenger, D. Lüthi, Q. Jiang, R. B. Smith, and R. Benoit, 2003: Structure and dynamics of an Alpine potential-vorticity banner. Quart. J. Roy. Meteor. Soc., 129, 825-855.

Scinocca, J. F., and N. A. McFarlane, 2000: The parametrization of drag induced by stratitified flow over anisotropic orography. Quart. J. Roy. Meteor. Soc., 126, 2353-2393.

Shutts, G. J., 1995: Gravity-wave drag parametrization over complex terrain: The effect of critical-level absorption in directional wind-shear. Quart J. Roy. Meteor. Soc., 121, 1005-1021.

_ 2003: Inertia-gravity wave and neutral Eady wave trains forced by directionally sheared flow over isolated hills. $J$. Atmos. Sci., 60, 593-606.

_ and A. Gadian, 1999: Numerical simulations of orographic gravity waves in flows which back with height. Quart. J. Roy. Meteor. Soc., 125, 2743-2765.

Smith, R. B., 1979: Some aspects of the quasi-geostrophic flow over mountains. J. Atmos. Sci., 36, 2385-2393.

_ 1984: A theory of lee cyclogenesis. J. Atmos. Sci., 41, 11591168.

- 1986: Further development of a theory of lee cyclogenesis. $J$. Atmos. Sci., 43, 1582-1602.

Speranza, A., A. Buzzi, A. Trevisan, and P. Malguzzi, 1985: A theory of deep cyclogenesis in the lee of the Alps. Part I: Modifications of baroclinic instability by localized topography. J. Atmos. Sci., 42, 1521-1535.

Vadas, S. L., D. C. Fritts, and M. J. Alexander, 2003: Mechanism for the generation of secondary waves in wave breaking regions. J. Atmos. Sci., 60, 194-214.

Webster, S., A. R. Brown, D. R. Cameron, and C. P. Jones, 2003: Improvements to the representation of orography in the Met Office unified model. Quart. J. Roy. Meteor. Soc., 129, 19892010. 